\title{
Benchmarking Cellulose Nanocrystals: From the Laboratory to Industrial Production
}

\author{
Michael S. Reid ${ }^{l}$, Marco Villalobos ${ }^{2}$ and Emily D. Cranston ${ }^{{ }^{*}}$ \\ ${ }^{1}$ Department of Chemical Engineering, McMaster University \\ Hamilton, Ontario, Canada, L8S 4L8 \\ ${ }^{2}$ Cabot Corporation, \\ Billerica, MA, US, 01821
}

*Corresponding author: ecranst@mcmaster.ca 


\begin{abstract}
:
The renewability, biocompatibility and mechanical properties of cellulose nanocrystals (CNCs) have made them an attractive material for numerous composite, biomedical and rheological applications. However, for CNCs to shift from laboratory curiosity to commercial applications, researchers must transition from CNCs extracted at the bench scale to material produced at an industrial scale. There are a number of companies currently producing kilogram to ton per day quantities of sulfuric acid-hydrolyzed CNCs, as well as other nanocelluloses, as described herein. With the recent intensification of industrially produced CNCs, the variety of cellulose sources, hydrolysis methods and purification procedures, characterization of these materials becomes critical. This has further been justified by the past two decades of research which demonstrate that CNC stability and behaviour is highly dependent on surface chemistry, surface charge density and particle size. This work outlines key test methods that should be employed to characterize these properties to ensure a "known" starting material and consistent performance. Of the sulfuric acid-extracted CNCs examined, industrially produced material compared well with laboratory-made CNCs, exhibiting similar charge density, colloidal and thermal stability, crystallinity, morphology and self-assembly behaviour. In addition, it was observed that further purification of CNCs, using Soxhlet extraction in ethanol, had minimal impact on nanoparticle properties and is unlikely to be necessary for many applications. Overall the current standing of industrially produced $\mathrm{CNCs}$ is positive suggesting that the evolution to commercial scale applications will not be hindered by CNC production.
\end{abstract}

\title{
Introduction
}

In line with the growing demand for sustainable and renewable energy, the desire for "green" materials has dramatically increased over the last decade. To meet this demand, materials that 
take inspiration from, or are directly extracted from natural resources, such as plants, mollusks, crustaceans, bacteria, and algae, have been the focus of a rapidly expanding field that seeks to include renewable materials in biomedical devices, ${ }^{1}$ energy storage applications, ${ }^{2-4}$ as rheological modifiers, ${ }^{5,6}$ reinforcing agents, ${ }^{7-10}$ replacements of petroleum-based polymers, ${ }^{11-14}$ and many other applications. ${ }^{15,16}$ Although renewable polymeric materials (e.g., cellulose, chitin, natural rubber) have been used for centuries, the recent "green movement" has pushed researchers to re-address many of these resources for new and innovative applications. In this light, renewable nanomaterials make up a quickly growing field with tremendous potential. Much like other nanomaterials (e.g., graphene, carbon nanotubes, nanoclays, metal and inorganic nanoparticles), renewable nanomaterials have high surface-area-to-volume ratios and unique nanoscale properties which are not present in macroscopic materials. ${ }^{17}$

As the Earth's most abundant polymer, cellulose and cellulose derivatives have a long tradition in industry and have been incorporated into countless products and processes. ${ }^{16}$ More recently, however, interest in cellulose has shifted towards nanoscale materials including cellulose nanofibrils (CNFs), bacterial nanocellulose and cellulose nanocrystals (CNCs). ${ }^{18}$ First reported by Nickerson and Habrle ${ }^{19}$ and subsequently imaged by Rånby and Ribi following acidic hydrolysis of cotton cellulose fibers, ${ }^{20} \mathrm{CNCs}$ have since been extracted from a number of other natural resources including, grasses, woods, algae, bacterial cellulose and tunicate. ${ }^{21}$ Composed of $\beta$ 1-4 linked D-glucose units, linear polymer chains are arranged in a highly crystalline cellulose I structure forming high aspect ratio particles that are insoluble in, but strongly bind water ${ }^{22}$ and depending on source and extraction procedure, range from $50-3000$ $\mathrm{nm}$ in length with cross sections of $3-20 \mathrm{~nm} .{ }^{23}$ The literature commonly presents CNCs as reinforcing agents in nanocomposites, rheological modifiers/stabilizers, and as additives in 
biomedical devices; these and many other applications are described in a number of comprehensive review papers. ${ }^{9,18,23-29}$ In addition to their high aspect ratio and large surface area, CNCs have unique physical properties, such as their ability to self-assemble into chiral nematic liquid crystalline phases, ${ }^{30-33}$ align in magnetic ${ }^{34-39}$ and electric fields, ${ }^{40-42}$ and exhibit piezoelectric reponsivity. ${ }^{43}$

Our work on CNCs has primarily focused on developing hybrid nanomaterials such as films, aerogels, and liquid formulated products. We often play on the synergy between auto-adhering polymers and surfactants with $\mathrm{CNCs}$ which enables superior stabilization and enhanced mechanical properties in emulsions, gels and foams. ${ }^{6,44-50}$ The surface modification routes we have developed are water-based and scalable leading to, for example, hydrophobic, responsive, biomimetic, and crosslinkable CNCs. ${ }^{51-55}$ Although we have targeted advanced applications such as industrial coatings, ${ }^{56,57}$ tissue scaffolds, ${ }^{6,48,50}$ energy storage ${ }^{2,58}$ water purification ${ }^{59}$ and food and cosmetics, ${ }^{44-47}$ we remain committed to thorough characterization of $\mathrm{CNC}$ particles and interfaces. This has included characterization of chemical, physical, mechanical and selfassembly properties, ${ }^{34,52,60-62}$ as well as the development of new methods to predict and assess CNC dispersion. ${ }^{63,64}$

Although CNCs have been extracted from a wide variety of natural cellulose sources using numerous methods, ${ }^{65} \mathrm{CNCs}$ obtained via sulfuric acid hydrolysis from cotton or wood have been the primary focus of both academia and industry. Commonly, CNCs are extracted by exposing cellulose to strong sulfuric acid ( $\sim 64 \mathrm{wt} \%)$, which favorably hydrolyzes accessible disordered regions leaving highly ordered cellulose in the form of rod-shaped particles. The strong acid hydrolysis additionally grafts anionic sulfate half esters $\left(\mathrm{OSO}_{3}{ }^{-}\right)$to the $\mathrm{CNC}$ surface, which electrostatically stabilize CNCs in aqueous environments. Significant research has focused on 
understanding and optimizing $\mathrm{CNC}$ extraction and it has been found that changes to the hydrolysis conditions can greatly affect particle dimensions, surface charge density and overall CNC yield. ${ }^{66,67}$

For example Dong et al. observed that higher temperatures and longer reaction times of hydrolysis of microcrystalline cellulose produced shorter CNCs with low aspect ratios. ${ }^{68}$ Similarly Beck et al. demonstrated that shorter, less polydisperse CNCs were produced from softwood pulps using longer hydrolysis times and increased acid-to-pulp ratios. ${ }^{69}$ More recently, Dong et al. studied the yield and sulfate half ester group density of CNCs extracted from softwood pulp by analyzing acid concentration, hydrolysis temperature and hydrolysis time using rotatable central composite experimental design. ${ }^{70}$ High acid concentrations $(>65 \mathrm{wt} \%)$ were found to produce CNCs with sulfate half ester content upwards of $391 \mathrm{mmol} / \mathrm{kg} \mathrm{CNCs}$, however at significantly reduced yield $(<20 \%)$. A maximum $\mathrm{CNC}$ yield of $66-69 \%$ with a sulfate half ester content of 241-265 mmol/kg CNC was achieved using 57-58 wt\% sulfuric acid at $64-67^{\circ} \mathrm{C}$ for 134-156 minutes. ${ }^{70}$ This is in excellent agreement with Wang et al. and Chen et al. who obtained similar results extracting CNCs from bleached kraft eucalyptus pulp. ${ }^{67,71}$

In addition to $\mathrm{CNC}$ dimensions, yield and sulfate half ester content, a recent publication by Bouchard et al. demonstrated that hydrolysis temperature significantly alters byproducts produced during hydrolysis and in turn, the CNC surface. ${ }^{72} \mathrm{CNCs}$ extracted from bleached softwood pulp at $45^{\circ} \mathrm{C}$ in $64 \mathrm{wt} \%$ sulfuric acid for $25 \mathrm{~min}$ were observed to contain nearly $12 \%$ by mass oligosaccharides which precipitate onto the CNC surface during the water quenching step of the hydrolysis reaction. These oligosaccharides, with a degree of polymerization between 7 and 20, significantly alter the rheological, colloidal and optical properties of the CNCs. Increasing the reaction temperature to $65^{\circ} \mathrm{C}$ reduced the degree of polymerization of 
oligosaccharide byproducts such that they remain soluble (and thus do not precipitate on CNC surfaces) following hydrolysis. CNCs produced without oligosaccharide layers were observed to be more viscous and less colloidally stable in high ionic strength environments. These studies emphasize that thorough understanding of $\mathrm{CNC}$ properties prior to use is critical as differences in particle dimension and charge density can greatly affect the rheological, ${ }^{73}$ colloidal, ${ }^{74}$ interfacial,$^{75}$ and reinforcing properties. ${ }^{76}$

Along with the hydrolysis conditions, post extraction treatments such as sonication or purification can further impact CNC properties and behaviour. Beck et al. showed that the pitch of chiral nematic phases could be controlled by varying the amount of sonication applied to CNC dispersions. ${ }^{77}$ High energy input was observed to increase the pitch of the chiral nematic phase and alter the reflected wavelength of dried CNC films. Similarly, Shafiei-Sabet et al. demonstrated that sonication reduced the shear viscosity of $\mathrm{CNC}$ dispersions at low shear rates by nearly two orders of magnitude. ${ }^{78}$ Interestingly the viscosity at high shear rates was largely unaffected by sonication. Changes to both the rheological and chiral nematic behaviour are currently not fully understood but are suggested to result from the liberation of excess charged ions from the particle surface following sonication or from the precipitated oligosaccharide layer. This liberation alters the electrostatic repulsion between particles and thus rheological behaviour and self-assembly. ${ }^{72}$

The influence of post extraction treatment was further demonstrated by Labet and Thielemans who showed that additional purification was needed to achieve consistent results when grafting polymers onto CNC surfaces. ${ }^{79}$ Soxhlet extraction of freeze dried CNCs in ethanol for $24 \mathrm{~h}$ not only improved the grafting density of $\varepsilon$-caprolactone by surface initiated ring opening polymerization but also the reproducibility. It is proposed that Soxhlet extraction removes small 
molecules, including xylobiose, 1,6-anhydroglucose and vanillic acid, as well as low molecular weight oligosaccharides, such as those observed by Bouchard et al. ${ }^{72}$ that precipitate onto the CNC surface during hydrolysis while leaving the bulk of the CNC structure intact. ${ }^{79}$ It is unclear however, to what degree byproducts can be removed via Soxhlet extraction as the solubility of oligosaccharides decreases in ethanol and thus would remain on the particle surface. ${ }^{80}$ Furthermore recent molecular dynamic simulations demonstrate that oligosaccharides strongly bind to crystalline cellulose surfaces, ${ }^{81}$ and that following sonication, oligosaccharides remain on the $\mathrm{CNC}$ surface. ${ }^{72}$

The various hydrolysis conditions, post extraction treatments, and diversity of cellulose starting material, highlight the need for thorough characterization of CNCs prior to use. Additionally it is critical that the community as a whole recognizes that CNCs produced from various sources and methods do not have the same properties and behaviour. (Note that this work does not investigate CNFs - there are many industrial producers in North America, Europe and $\operatorname{Japan}^{82,83}$ but comparing their materials is outside of the scope of this study.) This is particularly important as over the last decade researchers have begun to transition from CNCs traditionally made in the laboratory at the bench scale to CNCs that have been produced industrially. Ultimately this transition must occur if commercial applications of CNCs are to be realized. To date there has yet to be a thorough comparison of CNCs produced at the bench scale to CNCs produced commercially. Largely CNCs produced commercially operate using proprietary technology and often the exact cellulose source, extraction process and purification is not entirely evident. Furthermore, as new producers enter the market and as extraction processes and cellulose sources of current producers change, there is potential for batch-to-batch variability, which must be understood. The Canadian Standards Association has outlined test methods to 
assess cellulosic nanomaterial properties and current Standards and nomenclature guidelines are in preparation by ISO and TAPPI, however, there is yet to be direct comparison between industrial and lab scale CNCs. ${ }^{84}$ Therefore as the $\mathrm{CNC}$ community grows and industrially produced material becomes more prevalent, essential questions may be asked:

1) Are CNCs from various producers equivalent?

2) What are the most practical characterization methods that should be employed prior to using CNCs?

3) When is further purification, such as Soxhlet extraction, required?

4) If producers change scale or starting material, what kind of changes can be expected?

Herein we present the first detailed comparison of CNCs extracted by sulfuric acid hydrolysis at the bench scale to those produced commercially. We seek to thoroughly characterize currently available industrially produced $\mathrm{CNCs}$ and present the most practical methods and procedures to assess particle dimensions, thermal and colloidal stability, and charge density such that researchers can have a known starting material prior to including CNCs in products. Additionally we investigate how Soxhlet extraction, as a purification method, alters CNC properties in comparison to "as received" material. We present clear experimental details and procedures such that new researchers and students can confidently prepare and use CNCs.

\section{Experimental}

Materials. Whatman cotton ashless filter aid was purchased from GE Healthcare Canada. Sulfuric acid, was obtained from Caledon Laboratory Chemicals (Georgetown, ON, Canada). 
Anhydrous ethanol was purchased from Commercial Alcohols (Brampton, ON, Canada). Hydrogen peroxide and Whatman glass microfiber extraction thimbles were purchased from Sigma Aldrich (Oakville, ON, Canada). Polyallylamine hydrochloride $(\mathrm{PAH}, \mathrm{Mw}=120$ 000-200 $000 \mathrm{~g} / \mathrm{mol}$ ) was purchased from Polysciences. All chemicals were used as received. Water used was purified Type I water with a resistivity of $18.2 \mathrm{M} \Omega \cdot \mathrm{cm}$ (Barnstead NANOpure DIamond system, ThermoScientific, Asheville, NC).

CNC Samples. The CNCs investigated in this work were obtained from current industrial producers of sulfuric acid extracted CNCs operating at various industrial scales: CelluForce, Alberta Innovates Technology Futures (AITF) and the US Department of Agriculture (USDA) Forest Products Labs (FPL) (supplied by the University of Maine) and compared to CNCs produced in our lab. Additionally, nanocelluloses produced by less conventional methods from American Process Inc. and Blue Goose Biorefineries were obtained and investigated to compare with traditional CNCs. All sulfuric acid extracted CNCs samples were received in sodium form as either spray dried or freeze dried powders or as concentrated dispersions.

\section{CelluForce}

CelluForce, the world's largest producer of CNCs with ton/day capacity, uses the traditional 64 wt\% sulfuric acid hydrolysis (pioneered by Dr. Derek Gray's group of McGill University and scaled up at FPInnovations Pointe Claire, QC, Canada) to produce CNCs from bleached Kraft pulp. $^{31,37,68,85}$ Following hydrolysis $\mathrm{CNCs}$ are diluted, separated from residual acid and neutralized to sodium form and spray dried. 


\section{USDA Forest Products Lab (FPL)}

CNCs are produced from strip-cut dissolving pulp via $64 \mathrm{wt} \%$ sulfuric acid hydrolysis for $60 \mathrm{~min}$ at $45^{\circ} \mathrm{C}$ in an oxygen free atmosphere in the Madison, WI pilot facility with $10 \mathrm{~kg} /$ day capacity. Following hydrolysis, $\mathrm{CNCs}$ are diluted with reverse osmosis water and sodium chlorite is added to remove colour. Finally, acid is neutralized by the addition of sodium hydroxide. Material is fractionated and concentrated using a $20 \mu \mathrm{m}$ membrane and sold as freeze dried, spray dried powders, or dispersion. ${ }^{86}$ Material investigated in this work was received as a $10 \mathrm{wt} \%$ dispersion.

\section{Alberta Innovates Technology Futures (AITF)}

To investigate how changing the cellulose source and scale of production effects industrially produced CNCs properties, CNCs from two different cellulose sources, cotton and wood, were supplied by AITF. Cotton sourced CNCs were produced at the g/day capacity via traditional sulfuric acid hydrolysis and received as freeze dried powder (labeled AITF). Wood based CNCs were produced at the pilot scale (kg/day capacity) from softwood dissolving pulp using $63.5 \mathrm{wt} \%$ sulfuric acid hydrolysis at $45^{\circ} \mathrm{C}$ for $2 \mathrm{~h}$. The hydrolysis was quenched with reverse osmosis water and $\mathrm{CNCs}$ were neutralized to sodium form using $\mathrm{NaOH}$. Samples were received as in a $1.1 \mathrm{wt} \%$ dispersion.

Other Nanocelluloses. These materials are not strictly considered $\mathrm{CNC}$ or $\mathrm{CNF}$ according to the published CSA Standard ${ }^{84}$ (and TAPPI/ISO Standards which are currently in preparation) although they possess some favourable properties of both. These nanocelluloses produced by alternative methods to the typical sulfuric acid hydrolyzed $\mathrm{CNCs}$ were investigated. While there are a number of companies selling "other nanocelluloses" online we have chosen only two 
relatively large scale producers of nanocellulose as a demonstration of what non-sulfuric acid hydrolyzed CNCs may look like. This benchmarking study does not include bacterial cellulose and CNF, despite their widespread production; the same concerns exist for these materials as for CNCs but are not addressed here.

American Process Inc.

American Process Inc. (API) operates a pilot scale facility using a proprietary method, called AVAP which uses $\mathrm{SO}_{2}$ and ethanol along with mechanical treatment to liberate both CNFs and CNCs from a variety of biomass sources. ${ }^{87,88}$ BioPlus $^{\mathrm{TM}}$ Crystals were purchased as a $6 \mathrm{wt} \%$ aqueous gel and used as received.

\section{Blue Goose Biorefineries}

Blue Goose Biorefineries Inc. uses a transition metal catalyzed oxidative method to produce cellulose nanocrystals from a variety of biomass sources. ${ }^{89}$ The product tested for this study is BGB Natural ${ }^{\mathrm{TM}}$ received as a $7.4 \mathrm{wt} \%$ aqueous gel. This has a mixture of fibrillar cellulose and nanomaterial. Blue Goose has since introduced a new product, BGB Ultra ${ }^{\mathrm{TM}}$ which is claimed to have a more similar size distribution and crystallinity to acid hydrolyzed CNCs, but with carboxyl functional groups instead of sulfate half esters. BGB Ultra ${ }^{\mathrm{TM}}$ was not evaluated in this study.

Lab-made Cellulose Nanocrystal Preparation. CNCs (denoted "Lab-Made" in the subsequent text) were prepared in the laboratory by sulfuric acid hydrolysis, as previously described. ${ }^{56} 40 \mathrm{~g}$ of cotton Whatman ashless filter aid was processed in $700 \mathrm{~mL}$ of $64 \mathrm{wt} . \%$ sulfuric acid at $45^{\circ} \mathrm{C}$ 
for 45 min under continuous mechanical stirring. Following hydrolysis, the reaction was quenched by a 10 -fold dilution with $4^{\circ} \mathrm{C}$ water and excess acid was removed by multiple rinsing and centrifugation steps. The precipitate was then diluted and dialyzed against purified water until $\mathrm{pH}$ of the water from successive washes stabilized between 5-6 ( $\sim 2$ weeks). The suspension was probe sonicated (Sonifier 450, Branson Ultrasonics, Danbury, CT) continuously in an ice bath for 15 min three times at $60 \%$ output. The $\mathrm{CNC}$ suspensions were filtered through Whatman glass fiber filter paper and neutralized (converted to the sodium salt form) by the addition of a calculated amount of $1 \mathrm{mM} \mathrm{NaOH}$ (based on condutometric titrations). Prior to further characterization and processing, all nanocellulose material, lab made and received from industry, was redispersed in water and lyophilized to prevent any change to sulfate half ester content. ${ }^{90}$ For all subsequent analysis, CNCs were redispersed and thoroughly sonicated as needed.

Soxhlet Extraction. Cellulose material was purified via Soxhlet extraction as outlined by Labet and Thielemans. ${ }^{79}$ Glass microfiber thimbles were soaked in $95 \%$ ethanol between 3 and 5 hours and dried at $80^{\circ} \mathrm{C}$ prior to extraction. Freeze dried CNCs (ca. $0.5 \mathrm{~g}$ ) were placed in glass microfiber extraction thimbles and Soxhlet extracted with anhydrous ethanol for $24 \mathrm{~h}$. Following extraction material was dried at $80^{\circ} \mathrm{C}$ overnight and stored over desiccant.

Conductometric Titration. Sulfate half ester content (and thus indirectly surface charge) was measured by conductometric titrations as outlined previously. ${ }^{91,92}$ Critically, conductometric titrations must be performed on $\mathrm{CNCs}$ in acid form $\left(-\mathrm{OSO}_{3} \mathrm{H}\right)$, and thus lab-made and industrially produced $\mathrm{CNCs}$ (in sodium form, $-\mathrm{OSO}_{3} \mathrm{Na}$ ) were treated with ion exchange resin as outlined by Beck et al. ${ }^{92} 20-50 \mathrm{~mL}$ of ca. $0.5 \mathrm{wt} \% \mathrm{CNC}$ dispersions were prepared by 
redispersing and thoroughly sonicating both "as received" and Soxhlet extracted freeze dried CNCs. Dispersions were then passed through a $1.9 \mathrm{~cm}$ inner diameter glass fritted column containing $15 \mathrm{~cm}$ of thoroughly rinsed $(>1000 \mathrm{~mL}$ water) Dowex Marathon $\mathrm{C}$ hydrogen form strong acid cation exchange resin. Between each CNC sample the column was rinsed with 300 $\mathrm{mL}$ of water to remove residual cellulose material. Based on the average $-\mathrm{OSO}_{3}{ }^{-}$content of 251 $\mathrm{mmol} / \mathrm{kg} \mathrm{CNC}$ (determined by conductometric titration) a maximum exchange of $0.5 \mathrm{meq} \mathrm{Na}^{+}$ ions to $\mathrm{H}^{+}$ions was determined for the complete set of samples. This corresponds to less than $2 \%$ of the total ion exchange capacity of the column (40.6 meq). Following ion exchange conductometric titrations were performed by diluting $2.5-5 \mathrm{~mL}$ dispersion of ca. $0.5 \mathrm{wt} \% \mathrm{CNCs}$ in $80 \mathrm{~mL}$ in water. $1 \mathrm{~mL}$ of $100 \mathrm{mM} \mathrm{NaCl}$ solution was added to increase conductivity to a measureable range. The conductivity and $\mathrm{pH}$ were continuously measured throughout the titration while $100 \mu \mathrm{L}$ aliquots of standardized $2 \mathrm{mM} \mathrm{NaOH}$ was added over a period of $30-45$ minutes. The volume-corrected conductivity was plotted from which the equivalence point was determined by the intersection of least squares regressions from the positive and negative sloped regions. A representative curve is presented in Figure 1. Samples were measured in triplicate and the standard deviation is presented as the confidence interval. 


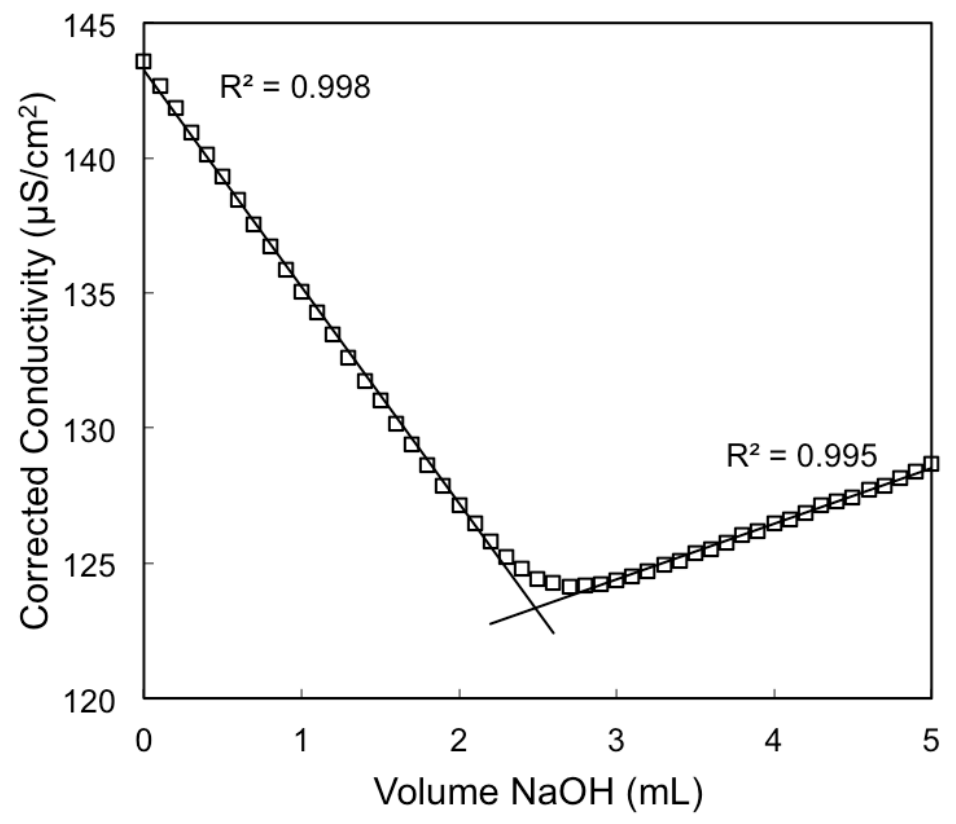

Figure 1: Representative conductometric titration curve of $\mathrm{CNCs}$ with $\mathrm{NaOH}$ showing the graphical determination of the equivalence point.

Zeta Potential. Zeta potential (related to surface potential and colloidal stability) of CNC samples was measured assuming Smoluchowski behaviour using a ZetaPlus electrophoretic mobility analyzer (Brookhaven Instruments Corp.). Note that some salt addition is necessary to get an accurate zeta potential measurement such that the double layer thickness around CNCs is not infinite and furthermore, suspensions which are unstable by eye (such as hydrophobically modified CNCs in water) will not give meaningful zeta potential readings. CNC dispersions of $0.25 \mathrm{wt} \%$ in $10 \mathrm{mM} \mathrm{NaCl}$ were prepared at $25^{\circ} \mathrm{C}$ and each sample was measured a total of 10 times, each measurement composed of 15 cycles. The confidence interval (error) presented is the standard deviation of samples measured in triplicate. 
Dynamic Light Scattering. Hydrodynamic "apparent particle size" was measured for 0.025 $\mathrm{wt} \% \mathrm{CNC}$ dispersions using a Malvern Zetasizer Nano particle analyzer at $20^{\circ} \mathrm{C}$ (no salt added). Note that if DLS measurements are taken on less concentrated samples multiple peaks and inaccurate data may appear due to the low scattering count but that at higher concentrations, particle agglomeration is promoted which may lead to larger apparent particle sizes. Each sample was measured 15 times and the average particle size distribution was obtained. The number average of each particle distribution was calculated and the confidence interval presented is the standard deviation of three separate samples.

X-ray Diffraction. XRD measurements, to obtain the degree of crystallinity, were performed on freeze dried CNCs samples using a Bruker D8 DAVINCI diffractometer (Bruker USA) with a cobalt sealed tube source $\left(\lambda_{\text {avg }}=1.79026 \AA\right), 35 \mathrm{kV}, 45 \mathrm{~mA}$ with a parallel focus Goebel Mirror, Vantec 500 area detector, and $0.5 \mathrm{~mm}$ micro-slit and $0.5 \mathrm{~mm}$ short collimator over a $2 \theta$ range of $8-45^{\circ}$. Si wafer blanks were subtracted from all sample measurements. Two dimensional area detector frames were integrated to produce diffraction patterns, which then underwent Rietveld refinement. Percent crystallinity was determined by deconvolution using the cellulose I single crystal information file (CIF) to define peak position and a fixed amorphous peak at $24.1^{\circ}$. A pseudo-Voigt function with linear background was used to fit peak shape and the CIF file with a March Dollase preferred orientation function model was used to fit peak intensity. For samples that contained both cellulose I and cellulose II, the percentage of each crystal phase is presented. It is important to note that there are a number of methods described in the literature used to determine the crystallinity of cellulose and is not only limited to XRD. ${ }^{93-96}$ Furthermore, the validity and limitations of these methods and data fitting routines is a hotly debated topic and is 
beyond the scope of this work. Largely XRD is reported and the Rietveld refinement is commonly considered to be the most accurate method. ${ }^{97}$ In a recent publication however, Ahvenainen et al. found good correlation with the five most common XRD fitting methods and the two dimensional Rietveld method. ${ }^{98}$ Moreover, within their work it is emphasized that comparison between samples and laboratories is extremely challenging. As such the crystallinity values presented here should be taken as relative (and comparable within this study) not absolute. Using the deconvolution method presented above, the error in these measurements is taken to be $3-5 \%$.

X-ray Photoelectron Spectroscopy. XPS spectra were collected to assess the surface elemental analysis of CNCs using a Physical Electronics (PHI) Quantera II spectrometer equipped with a monochromatic Al K- $\alpha$ X-ray $(1486.7 \mathrm{eV})$ source operated at $50 \mathrm{~W}$ and $15 \mathrm{kV}$. The system base pressure was no higher than $1.0 \times 10^{-9}$ Torr, with an operating pressure that did not exceed 2.0 $\mathrm{x} 10^{-8}$ Torr. A pass energy of $280 \mathrm{eV}$ was used to obtain all survey spectra and $26 \mathrm{eV}$ was used for carbon high resolution data and $55 \mathrm{eV}$ for all other high resolution data. High resolution $\mathrm{C}$ spectra allow for distinction between the various bonding environments of carbon atoms within the sample. Excluding hydrogen, the carbon bonding environments within cellulose are: carbon bound to solely carbon via single bonds, $\mathrm{C}-\mathrm{C}(\mathrm{C} 1)$, carbon bound to oxygen via a single bond $\mathrm{C}-$ $\mathrm{O}(\mathrm{C} 2)$ and carbon bound to two oxygen via single bonds $\mathrm{O}-\mathrm{C}-\mathrm{O}(\mathrm{C} 3)$.

All spectra were obtained at $45^{\circ}$ take off angles, and a dual beam charge compensation system was used for neutralization of all samples. The instrument was calibrated using a sputtercleaned $\mathrm{Ag}$ surface, where the $\mathrm{Ag} 3 \mathrm{~d}_{5 / 2}$ peak had a binding energy of $368.3 \pm 0.1 \mathrm{eV}$ and full width at half maximum for the $\mathrm{Ag} 3 \mathrm{~d}_{5 / 2}$ peak was at least $0.52 \mathrm{eV}$. Data manipulation was 
performed using PHI MultiPak Version 9.4.0.7 software. Measurements were performed on freeze dried material adhered to an aluminum alloy substrate with double sided tape .

Atomic Force Microscopy. AFM was used to measure the dimensions of CNCs and calculate the size polydispersity. Submonolayer CNC films were prepared on polished silicon wafers (MEMC Electronic Materials Sdn Bhd, Petaling Jaya, Malaysia) by spin coating $0.01 \mathrm{wt} \% \mathrm{CNC}$ suspensions under $\mathrm{N}_{2}$ gas (G3P Spincoat, Specialty Coating Systems Inc. Indianapolis, USA) at $4000 \mathrm{rpm}$ for $30 \mathrm{~s}$ with a $7 \mathrm{~s}$ acceleration ramp. Silicon substrates were cleaned in a piranha solution ( $3: 1$ concentrated sulfuric acid to hydrogen peroxide) for $30 \mathrm{~min}$, followed by continuous rinsing with purified water and drying with $\mathrm{N}_{2}$ gas. Suspensions of $0.01 \mathrm{wt} \%$ were prepared by dispersing freeze dried CNCs in water and sonicating at $60 \%$ amplitude for $30 \mathrm{~s}$. To reduce $\mathrm{CNC}$ aggregation on the surface, silicon substrates were spin coated with a precursor layer of $0.1 \mathrm{wt} \%$ cationic $\mathrm{PAH}$ solution and rinsed (by spin coating water) prior to $\mathrm{CNC}$ deposition.

AFM images were collected in alternating current (AC) mode using an Asylum MFP-3D instrument (Asylum Research an Oxford Instrument Company, Santa Barbara, CA). Images were collected in air under ambient conditions using rectangular FMR cantilevers (NanoWorld) with normal spring constants of $1.2-5.5 \mathrm{~N} / \mathrm{m}$ and resonant frequencies of $60-90 \mathrm{kHz}$. Images were processed in Igor Pro 6.0 running Asylum Research AFM software (version 13.17) using a second order flatten routine. CNC dimensions presented (cross section and length) were obtained from the analysis of a minimum of 100 particles. Confidence intervals presented are the standard deviation of the average particle dimensions measured. 
Thermal Gravimetric Analysis. Thermal gravimetric analysis (TGA) was performed using TA Instruments Q50 thermal analyzer under a constant $100 \mathrm{~mL} / \mathrm{min}$ argon flow. A minimum of $1 \mathrm{mg}$ of freeze-dried as received and Soxhlet extracted CNCs were heated to $600^{\circ} \mathrm{C}$ at heating rate of $10^{\circ} \mathrm{C} / \mathrm{min}$.

Liquid Crystalline Self-Assembly. The phase separation of CNC suspensions above a critical concentration of ca. $4.5 \mathrm{wt} \%$ into a lower chiral nematic liquid crystalline phase and an upper isotropic phase has been well documented and furthermore implies well dispersed individualized

CNCs. ${ }^{30,37,68,99}$ To test for this behavior, $50 \mathrm{~mL}$ of $1 \mathrm{wt} \% \mathrm{CNC}$ suspensions were prepared from freeze dried as received material. Samples were thoroughly sonicated and filtered through glass microfibre filter paper and allowed to gently evaporate to ca. $5 \mathrm{~mL}$ under constant stirring in ambient conditions (ca. 1 week). Samples were transferred to flat capillary tubes (inner dimensions $10 \times 1 \mathrm{~mm}$ ) and allowed to reach equilibrium over 30 days. Photos were taken between crossed polarizers to demonstrate phase separation. The chiral nematic texture of the anisotropic phase was imaged by polarized optical microscopy taken at 10 fold magnification using a Nikon Eclipse LV100POL microscope.

\section{Results}

$\mathrm{CNC}$ behaviour depends on surface charge density, crystallinity and particle dimensions. Understanding and characterizing $\mathrm{CNC}$ properties is critical as, cellulose source, hydrolysis conditions, and post extraction treatment impact these properties and ultimately determine suitable applications for CNCs. As the $\mathrm{CNC}$ community expands, thorough characterization is crucial, particularly as industrially produced $\mathrm{CNCs}$, which use proprietary technology, are 
beginning to replace traditionally lab made CNCs. Below we compare the properties of sulfuric acid extracted CNCs produced industrially and at the lab scale. Furthermore, we explore the effect of Soxhlet extraction and present the most practical methods to assess surface charge density, colloidal and thermal stability, particle dimensions and crystallinity.

\section{Sulfate Half Ester Content}

The sulfate half ester $\left(\mathrm{OSO}_{3}{ }^{-}\right)$content of $\mathrm{CNCs}$ extracted by sulfuric acid hydrolysis was measured by conductometric titration following dialysis and strong acid ion exchange resin treatment; the values ranged from $206-335 \mathrm{mmol} / \mathrm{kg} \mathrm{CNC}$ in agreement with literature, ${ }^{92}$ as presented in Figure 2. This corresponds to $0.66-1.1 \% \mathrm{~S}, 0.37-0.61$ charges $/ \mathrm{nm}^{2}$ or about 1 sulfate half ester for every $2-3$ surface anhydroglucose units assuming the dimensions of LabMade CNCs reported below. The broad range of $\mathrm{OSO}_{3}{ }^{-}$contents of $\mathrm{CNCs}$ extracted from the same process highlights the need for careful characterization of all material prior to use. Following Soxhlet extraction in ethanol for $24 \mathrm{~h}$, only minor changes in $\mathrm{OSO}_{3}{ }^{-}$content were observed with the largest change being a $7.5 \%$ decrease in surface charge measured for FPL CNCs. Interestingly, the sulfate content increased for CNCs from CelluForce whereas it decreased for Lab-Made, AITF and FPL CNCs. As Soxhlet extraction is thought to remove residual oligomers from the particle surface and other hydrocarbon impurities, ${ }^{79}$ formerly inaccessible $\mathrm{OSO}_{3}{ }^{-}$groups may become accessible and increase the apparent sulfate half ester content. Conversely, if the adsorbed oligomers themselves are sulfated (which is likely), removal would decrease the overall sulfate half ester content. 


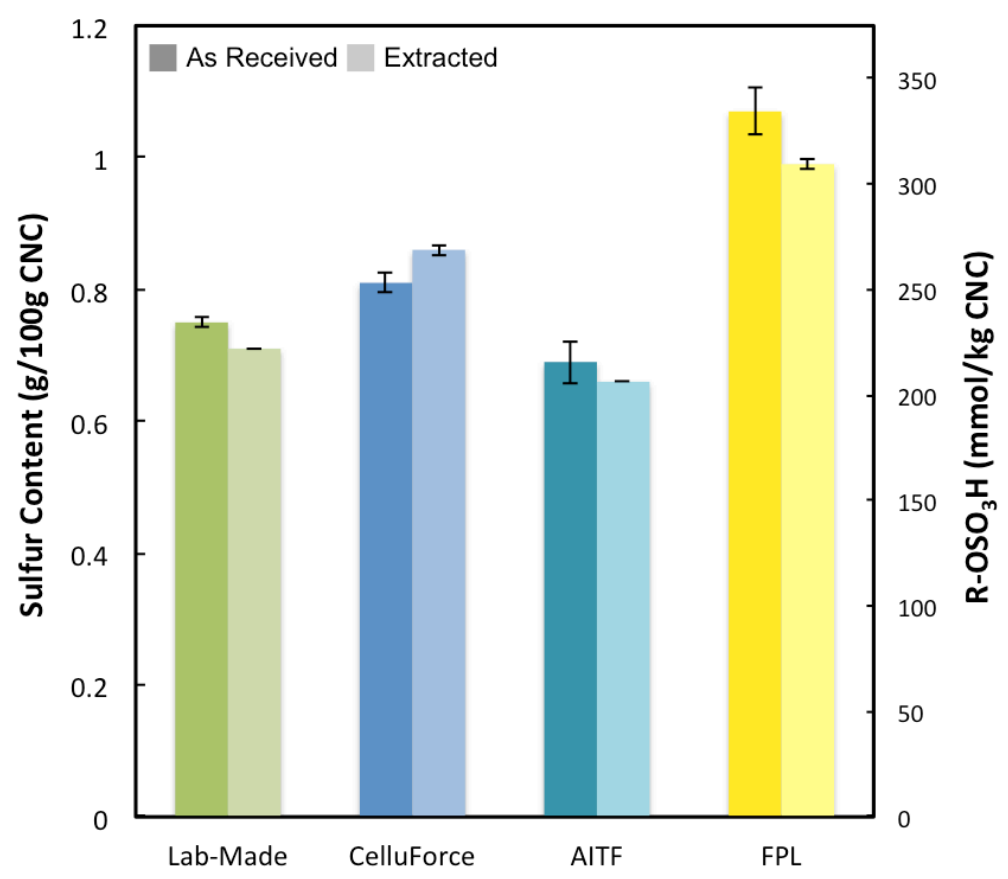

Figure 2: Sulfur content (left axis) and sulfate half ester content (right axis) for CNCs "as received" (which in this case includes dialysis and ion exchange resin treatment) and after Soxhlet extraction, as determined by conductometric titrations.

\section{Colloidal Stability}

The colloidal stability of lab and industrially produced CNCs was assessed by zeta potential calculations from electrophoretic mobility measurements. CNCs are well known to be electrostatically stabilized by anionic sulfate half ester groups grafted to the particle surface and thus measuring the zeta potential provides significant insight into the behaviour of CNCs in aqueous environments. Moreover, following CNC surface modification, the zeta potential is one of the primary tools used to measure the degree of modification particularly with polymer grafting. A reduction in the (absolute value of the) potential is attributed to modification either at the ester group or other $\mathrm{OH}$ groups, which make $\mathrm{OSO}_{3}{ }^{-}$groups less accessible. ${ }^{46,52}$ Figure 3 
presents the zeta potential of $\mathrm{Na}^{+}$form CNCs as received and following Soxhlet extraction. It is important to note that when calculating the zeta potential using the Smoluchowski or Hückel equation that assumptions are made about particle shape and behaviour in an electric field. It is perhaps more accurate to report electrophoretic mobility, however zeta potential is most often presented in the literature thus is reported here. All CNCs display zeta potential values less than $35 \mathrm{mV}$, which is significantly less than $-20 \mathrm{mV}$, typically assumed to be the maximum (negative value) required for moderate colloidal stability. ${ }^{100}$ Following Soxhlet extraction, CNCs remain stable and only a small $(<5 \mathrm{mV})$ increase is observed for Lab-Made, AITF and FPL CNCs. This small increase in zeta potential corroborates well with the small decrease in sulfate half ester content by conductometric titration.

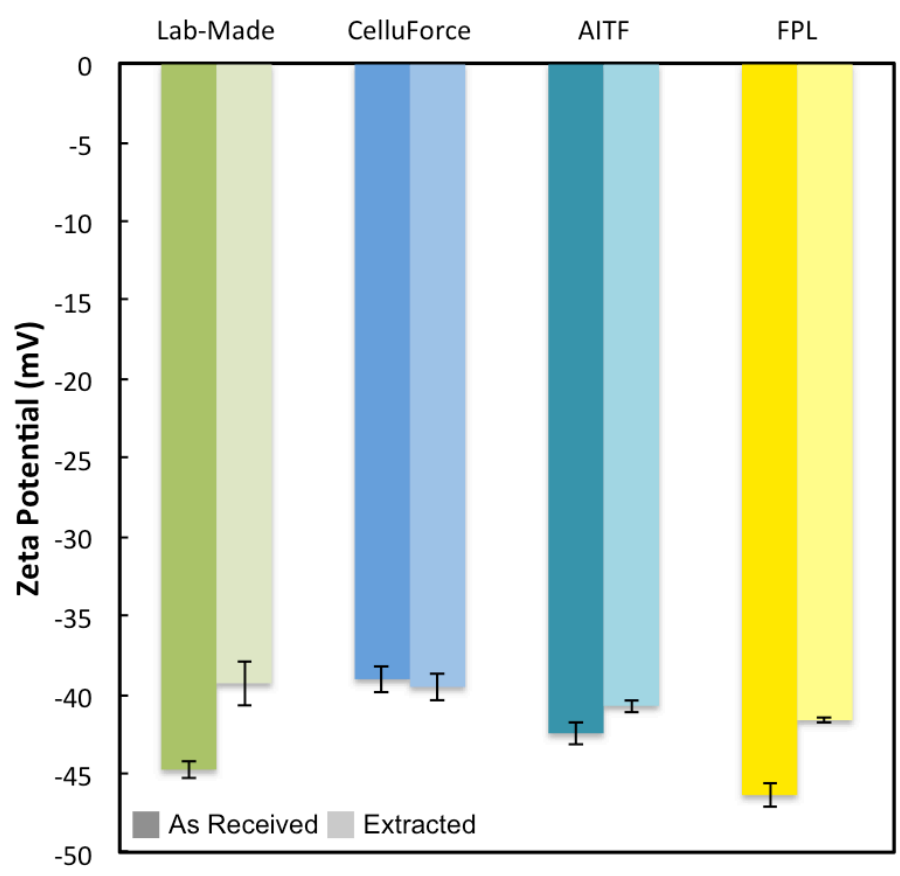

Figure 3: Zeta potential for "as received" and Soxhlet extracted CNCs in a $0.25 \mathrm{wt} \%$ dispersion with $10 \mathrm{mM} \mathrm{NaCl}$. 


\section{Particle Size and Morphology}

A significant portion of particle behaviour can be attributed to particle shape and morphology. For example, CNCs are ideal reinforcing materials since high aspect ratio particles reduce the volume percentage required to reach the percolation threshold. ${ }^{101}$ Similarly, shear thinning behaviour results from the high aspect ratio particles aligning with flow. ${ }^{78}$ Figure 4 presents the apparent particle size of $\mathrm{Na}^{+}$-form CNCs before and after Soxhlet extraction. Critically, DLS monitors the scattering intensity from particles undergoing Brownian motion and determines particle size via the Stokes-Einstein relation. However, the Stokes-Einstein relation assumes that the diffusion coefficient is constant which is not the case for rod shaped particles, such as CNCs, as the diffusion constants perpendicular and parallel to the particle axis differ. As a result, particle sizes measured by DLS are not absolute. Nonetheless DLS is an effective tool for measuring, relative particle sizes and the state of dispersion of particles (if the same instrument, sample preparation and protocol is used).

Figure 4 shows that $\mathrm{CNCs}$ from the various producers range in apparent particle diameter from $55-85 \mathrm{~nm}$. Particle sizes less than $100 \mathrm{~nm}$ are typical of CNCs measured by DLS and result from the assumptions discussed above. ${ }^{102}$ Largely, particle size did not significantly change following Soxhlet extraction with the only statistical difference observed for AITF CNCs. The increased particle size observed for AITF CNCs is likely due to particle agglomeration during Soxhlet extraction as no swelling is expected to occur. ${ }^{103}$ Exposing CNCs to unsuitable solvents force CNCs to strongly aggregate and increase cohesive particle-particle interactions. ${ }^{63}$ Potentially sonication following Soxhlet extraction was insufficient to completely redisperse CNCs resulting in CNCs with larger apparent sizes. 


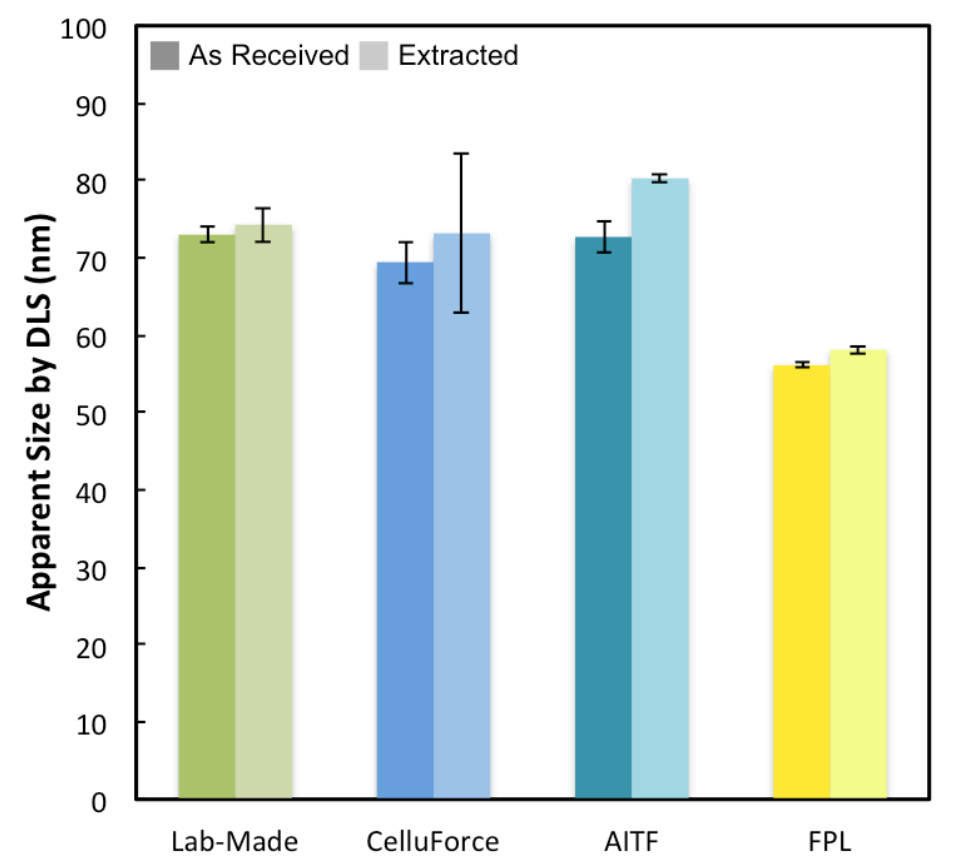

Figure 4: Apparent particle size as determined by DLS for "as received" and Soxhlet extracted CNCs in a $0.025 \mathrm{wt} \%$ dispersion.

Although DLS provides an assessment of the relative particle size and colloidal stability, particle dimensions are more appropriately measured by microscopy, specifically transmission electron microscopy (TEM) or AFM (or a combination of DLS and microscopy ${ }^{102}$ ). Both microscopy techniques require significantly more time and sample preparation than DLS yet the length and cross section of individual CNCs can be measured directly and meaningful statistical data can be obtained. ${ }^{104,105}$ Figure 5 displays representative $5 \mu \mathrm{m} \times 5 \mu \mathrm{m}$ AFM images of CNCs from each producer from which rod shaped, high aspect ratio particles are visible. No evidence of macroscopic material was observed in any CNC samples.

CNC dimensions were determined by selectively measuring the length and cross section of a minimum of 100 individual particles. No particle below $20 \mathrm{~nm}$ was measured as tip effects 
convolute particle dimensions (Figure 6). ${ }^{106}$ Similarly, aggregated CNCs were not measured since the exact boundaries of individual CNCs could not be distinguished. Finally, the cross section of the CNCs is taken as the height of the $\mathrm{CNC}$ at the centre of the particle to avoid tip broadening effects. The average values, including the aspect ratio, are presented in Table 1. All CNC samples exhibit a relatively narrow distribution of lengths, with the majority of particles between 100-200 nm. CelluForce CNCs shows the widest distribution with particles that range from $60-500 \mathrm{~nm}$ in length. Surprisingly the length and cross section of CNCs produced by LabMade, AITF, CelluForce and FPL were statistically equivalent yet the apparent size measured by DLS suggested that FPL CNCs were smaller. This emphasizes the need to completely characterize all CNCs prior to use and that no single characterization method can truly access particle behaviour and properties. The cross sectional analysis of the CNCs shows comparable distribution however, the CNCs produced from wood sources (CelluForce and FPL) have slightly narrower particles leading to larger average aspect ratios, as expected. ${ }^{69}$

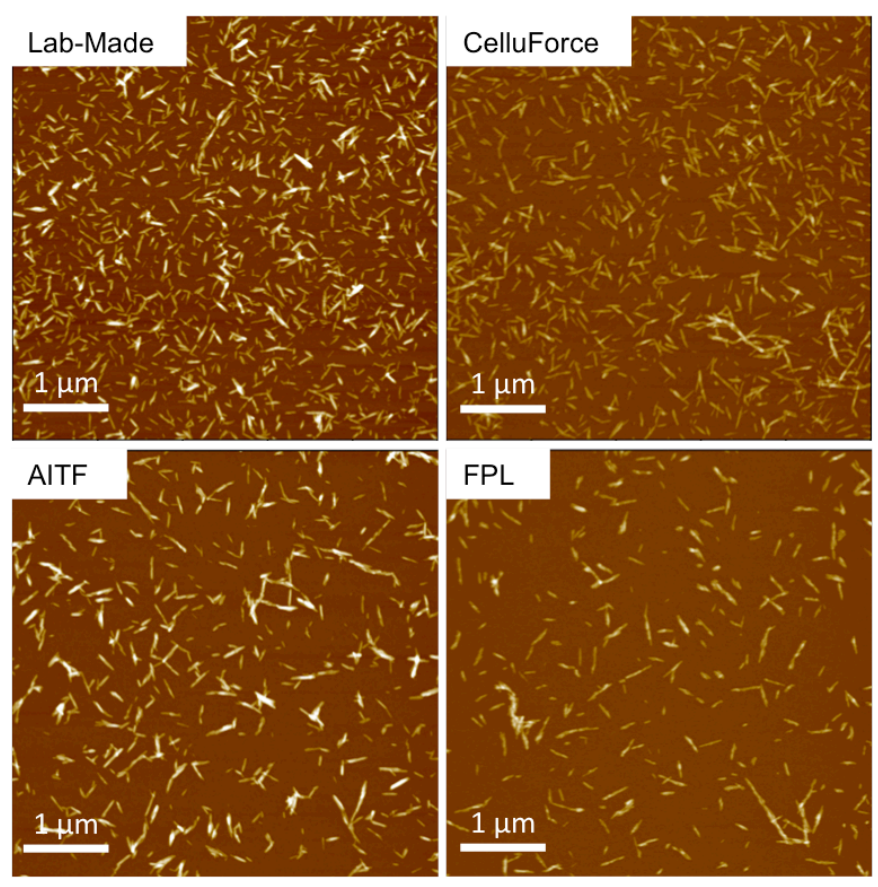

Figure 5: AFM height images of "as received" CNCs from various producers. 

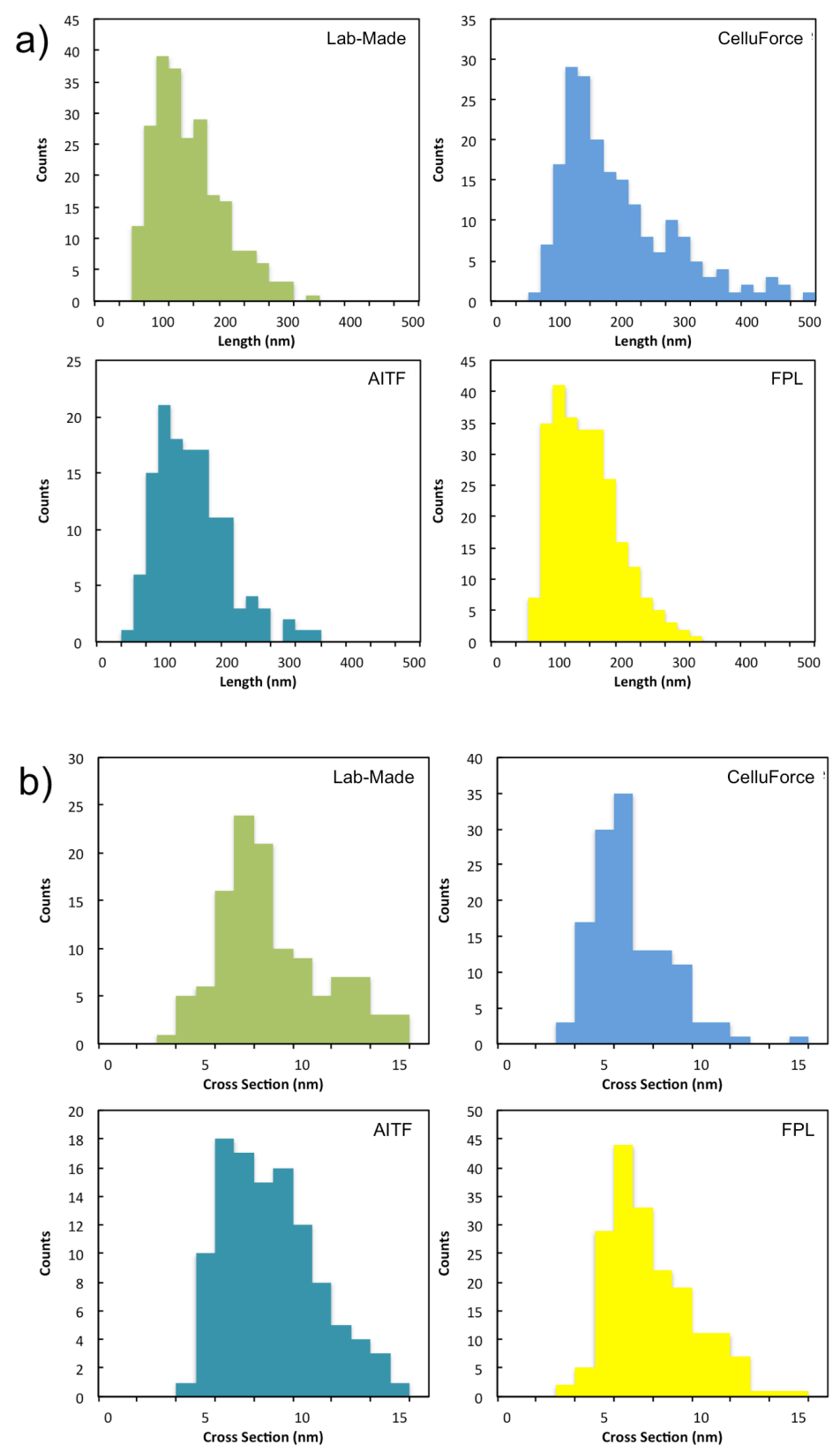

Figure 6: Particle length (a) and cross section (b) distributions of CNCs from various producers as measured by AFM. 
Table 1: Average length, cross section and aspect ratios of CNCs measured by AFM

\begin{tabular}{lccc} 
& Length $(\mathrm{nm})$ & Cross Section $(\mathrm{nm})$ & Aspect Ratio \\
\hline \hline Lab-Made & $132 \pm 55$ & $8 \pm 3$ & 16 \\
CelluForce & $183 \pm 88$ & $6 \pm 2$ & 31 \\
AITF & $134 \pm 56$ & $8 \pm 2$ & 17 \\
FPL & $134 \pm 52$ & $7 \pm 2$ & 19
\end{tabular}

\section{Crystallinity}

The crystallinity of each CNC sample was assessed by XRD and is presented in Table 2. Representative diffraction patterns are located in the Supporting Information, Figures S1-S4. We again note that the appropriate method for determining crystallinity of CNCs is a debated topic and that the values presented here are relative. CNCs from Lab-Made, CelluForce and AITF exhibited purely cellulose I structure with total crystallinity ranging from $88-94 \%$ in reasonable agreement with literature. ${ }^{23,51}$ Uniquely, CNCs from FPL could not be fit solely to cellulose I structure, consistent with previous studies, ${ }^{107}$ and thus cellulose II was included to determine the percentage of each polymorph. The resulting fits show that FPL CNCs contain nearly equal percentages of cellulose I and cellulose II. Currently, it is unclear why FPL CNCs contain a significant portion of cellulose II, it could be present in the starting material, or it could result from exposure to high concentrations of $\mathrm{NaOH}$ prior to or following hydrolysis. Concentrated $\mathrm{NaOH}$ is known to dissolve cellulose I, such as in mercerization, ${ }^{108}$ and the resulting dissolved cellulose polymer can precipitate onto the CNC surface in the more thermodynamically favourable structure of cellulose II. No significant change in crystallinity following Soxhlet extraction was observed for CNCs containing solely cellulose I which is to be expected since cellulose I does not swell or dissolve in ethanol. ${ }^{63}$ Conversely, FPL CNCs show a significant 
change in polymorph composition with the percentage of cellulose I nearly three times greater than cellulose II after Soxhlet extraction and suggesting that a portion of loosely bound material, which potentially contains cellulose II was lost.

Table 2: Percent crystallinity determined by XRD of CNCs "as received" and after Soxhlet extraction.

\begin{tabular}{lcccc} 
& \multicolumn{2}{c}{ As Received } & \multicolumn{2}{c}{ Soxhlet Extracted } \\
\cline { 2 - 5 } Cellulose Structure & I & II & I & II \\
\hline \hline Lab-Made & 93.4 & - & 91.4 & - \\
CelluForce & 89.9 & - & 88.7 & - \\
AITF & 91.2 & - & 91.8 & - \\
FPL & 53.9 & 46.1 & 74.2 & 25.8
\end{tabular}

\section{Chemical Composition}

The chemical composition of the CNCs was analyzed using XPS. Although cellulose was one of the early surfaces probed by XPS (formally referred to as $\operatorname{ESCA}^{109}$ ), the ability to quantify each "type" of carbon without contamination obscuring results remains challenging. ${ }^{110}$ Theoretically, cellulose is an excellent material for analysis since it contains only oxygen and carbon in an O/C ratio of 0.83 . Furthermore, all carbon is bound to at least one oxygen and thus only $\mathrm{C} 2$ and $\mathrm{C} 3$ should be present (see Experimental for description of carbon type labels). ${ }^{111}$ However, C1 carbon (C-C-C or C-C-H) is notoriously difficult to remove and is nearly always present in all measurements, even on clean gold surfaces. ${ }^{112}$ Largely attributed to hydrocarbon contamination, "clean" cellulose sources contain 5-7\% C1 carbon, making absolute comparison of cellulose 
carbon contents extremely difficult, particularly between different research labs and equipment. The problem is further amplified by sample inhomogeneity, charging, and degradation/redeposition of degraded products during measurements. Nonetheless XPS is a useful technique in determining relative changes at the particle surface, particularly when modifications contain noncarbon elements. ${ }^{113}$ More recently Labet and Thielmans used XPS to characterize CNCs before and after Soxhlet extraction in an effort to improve the reproducibility of CNC surface modification. ${ }^{79}$ The quantity of $\mathrm{C} 1$ was used as a measure of non-cellulosic material on the $\mathrm{CNC}$ surface. While $\mathrm{C} 1$ contamination from the environment makes it difficult to determine the exact carbon percentages, a decrease of ca. $10 \%$ was observed following extraction in their work. ${ }^{79}$

Table 3 presents the carbon percentages and $\mathrm{O} / \mathrm{C}$ ratios for $\mathrm{CNCs}$ as received and after Soxhlet extraction. All samples were measured under the same conditions and we emphasize that these are relative values and that environmental contamination and charging can significantly alter percentages. Clearly, the $\mathrm{C} 1$ content between samples varies greatly and is larger than the accepted range of $5-7 \%$ for clean cellulose surfaces, suggesting that indeed there is contamination on the $\mathrm{CNC}$ surface. The source of this contamination is difficult to determine as the environmental conditions in which industry $\mathrm{CNCs}$ are produced is not fully known. One potential source is the containers CNCs are shipped in since plastic containers are known to impart $\mathrm{C} 1$ carbon. ${ }^{110}$ The $\mathrm{O} / \mathrm{C}$ ratio for all $\mathrm{CNCs}$ is below the theoretical value of 0.83 , again supporting the presence of non-cellulosic material. After Soxhlet extraction, the relative $\mathrm{C} 1$ percentages decrease for Lab-Made, CelluForce and AITF CNCs and remain nearly constant for FPL CNCs. Although the Soxhlet extraction reduces the overall $\mathrm{C} 1$ content, the effect on the $\mathrm{O} / \mathrm{C}$ ratio is less clear. Lab-Made CNCs increase to 0.87 , higher than the theoretical value, where as CelluForce CNCs exhibit a decrease to 0.73 . Most notably are FPL CNCs, which show a 
dramatic reduction in $\mathrm{O} / \mathrm{C}$ ratio and is potentially due the loss of cellulosic material in the form of cellulose II during extraction.

Table 3: Carbon composition determined by XPS of CNCs "as received" and after Soxhlet extraction along with oxygen to carbon ratio.

\begin{tabular}{|c|c|c|c|c|c|c|c|c|}
\hline & \multicolumn{6}{|c|}{ Composition Percentage } & \multirow{2}{*}{\multicolumn{2}{|c|}{$\mathrm{O} / \mathrm{C}$}} \\
\hline & \multicolumn{2}{|c|}{ C1 } & \multicolumn{2}{|c|}{$\mathrm{C2}$} & \multicolumn{2}{|c|}{ C3 } & & \\
\hline & $\begin{array}{c}\text { As } \\
\text { Received }\end{array}$ & $\begin{array}{c}\text { Soxhlet } \\
\text { Extracted }\end{array}$ & $\begin{array}{c}\text { As } \\
\text { Received }\end{array}$ & $\begin{array}{c}\text { Soxhlet } \\
\text { Extracted }\end{array}$ & $\begin{array}{c}\text { As } \\
\text { Received }\end{array}$ & $\begin{array}{c}\text { Soxhlet } \\
\text { Extracted }\end{array}$ & $\begin{array}{c}\text { As } \\
\text { Received }\end{array}$ & $\begin{array}{c}\text { Soxhlet } \\
\text { Extracted }\end{array}$ \\
\hline Lab-Made & 13.9 & 6.1 & 44.6 & 54.1 & 41.6 & 39.8 & 0.77 & 0.87 \\
\hline CelluForce & 18.4 & 13.8 & 57.8 & 61.8 & 23.9 & 17.5 & 0.77 & 0.73 \\
\hline AITF & 21.8 & 15.2 & 42.3 & 58.8 & 35.9 & 26 & 0.74 & 0.76 \\
\hline FPL & 11.2 & 11.4 & 69.2 & 67.9 & 18.5 & 20.3 & 0.77 & 0.57 \\
\hline Theoretical & 0 & - & 83.3 & - & 16.7 & - & 0.83 & - \\
\hline
\end{tabular}

Thermal Stability. The thermal stability of industrially produced and lab made CNCs was investigated by TGA of freeze-dried material. CNCs showed typical thermal degradation behaviour ${ }^{107,114}$ and good stability with all "as received" material maintaining at least $95 \%$ mass above $250^{\circ} \mathrm{C}$ (Figure 7). The most significant difference can be seen in the char residue mass percentages $\left(500^{\circ} \mathrm{C}\right)$, which range from 11 to $27 \%$ for Lab-Made and AITF CNCs being the lowest and highest, respectively. The composition of the char residue is currently unclear but is proposed to contain levoglucosan and low molecular weight hydrocarbons along with hydrogen, $\mathrm{CO}, \mathrm{CO}_{2}$ and $\mathrm{H}_{2} \mathrm{O} \cdot{ }^{107}$ Following Soxhlet extraction, thermal stability of Lab-Made, CelluForce and FPL CNCs remained unchanged (see supporting Information Figures S5-S7). Conversely, 
Soxhlet extracted AITF CNCs showed reduced thermal stability with 95\% mass maintained only until $237^{\circ} \mathrm{C}$ (see Supporting Information Figure S8).

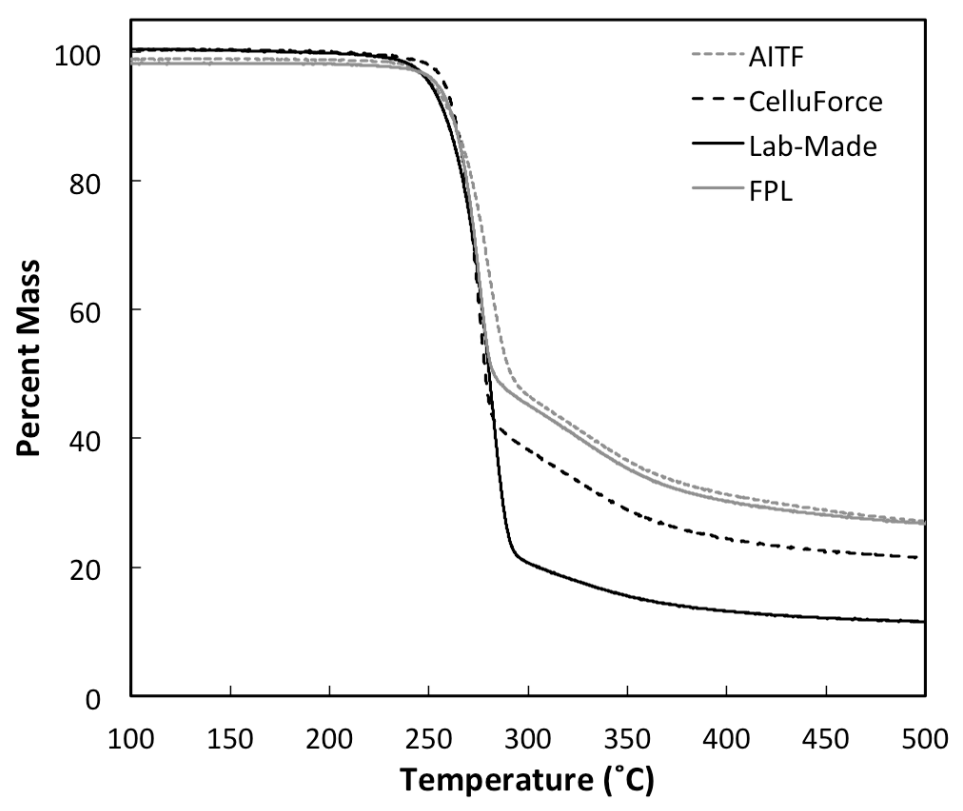

Figure 7: TGA curves of freeze dried "as received" lab made and industrially produced CNCs.

\section{Liquid Crystalline Properties and Self-Assembly}

The self-assembly and formation of chiral nematic phases in CNC suspensions can be used as an indicator for good particle behaviour. The exact onset of phase separation can indicate average $\mathrm{CNC}$ size (or lack of aggregation), size distribution, surface charge and ionic strength of the suspension. ${ }^{32,37,68}$ Figure 8 shows (a) CNC suspensions of "as received" samples at $1 \mathrm{wt} \%$ (below onset of phase separation) which shows slight variation in translucency which is affected primarily by average particle size and degree of agglomeration, and (b) concentrated $\mathrm{CNC}$ suspensions (above the onset of phase separation) after at least 10 days of self-assembly. Clear separation between the anisotropic and isotropic phases can be seen in all samples. Different volume fractions of the two phases are due to varying concentrations (some material is lost 
during filtration), charge density and aspect ratio of the particles. All CNCs investigated show the expected phase separation behaviour implying good dispersion of nanoparticles and minimal contamination, which would interfere with self-assembly (salts, sugars, polymers) in the "as received" samples.

Notably CelluForce CNCs exhibited phase separation at significantly lower concentrations $(3.5 \mathrm{wt} \%)$ whereas the others were all above $7 \mathrm{wt} \%$. This is likely due to the higher aspect ratio of the particles (see Table 1) similar to what has been observed in sulfuric acid extracted bacterial cellulose. ${ }^{115}$ At higher concentrations, CelluForce dispersions were observed to be completely anisotropic and kinetically arrested, ${ }^{116}$ showing, no biphasic behaviour. The anisotropic phase was investigated by polarized optical microscopy (POM) to confirm the presence of chiral nematic phases; Figure 9 shows that all samples display the characteristic fingerprint texture of chiral nematic liquid crystals. Due to the difference in concentrations between samples, an analysis of pitch length is not particularly relevant but generally agrees with the literature. 


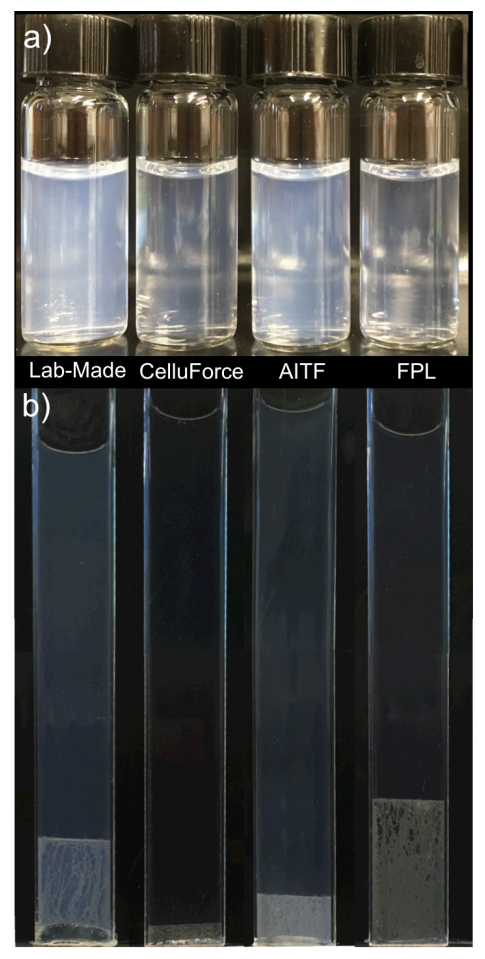

Figure 8: a) Photographs of $1 \mathrm{wt} \%$ suspensions of "as received" CNC samples. (b) Concentrated CNC suspensions: Lab-Made $8 \mathrm{wt} \%$, CelluForce $3.5 \mathrm{wt} \%$, AITF $8 \mathrm{wt} \%$, and FPL $7 \mathrm{wt} \%$, after 30 days in rectangular capillary tubes imaged between cross polarizers. Note: Due to sealing the capillary, the $\mathrm{CNC}$ dispersion does not reside directly at the bottom of the capillary.

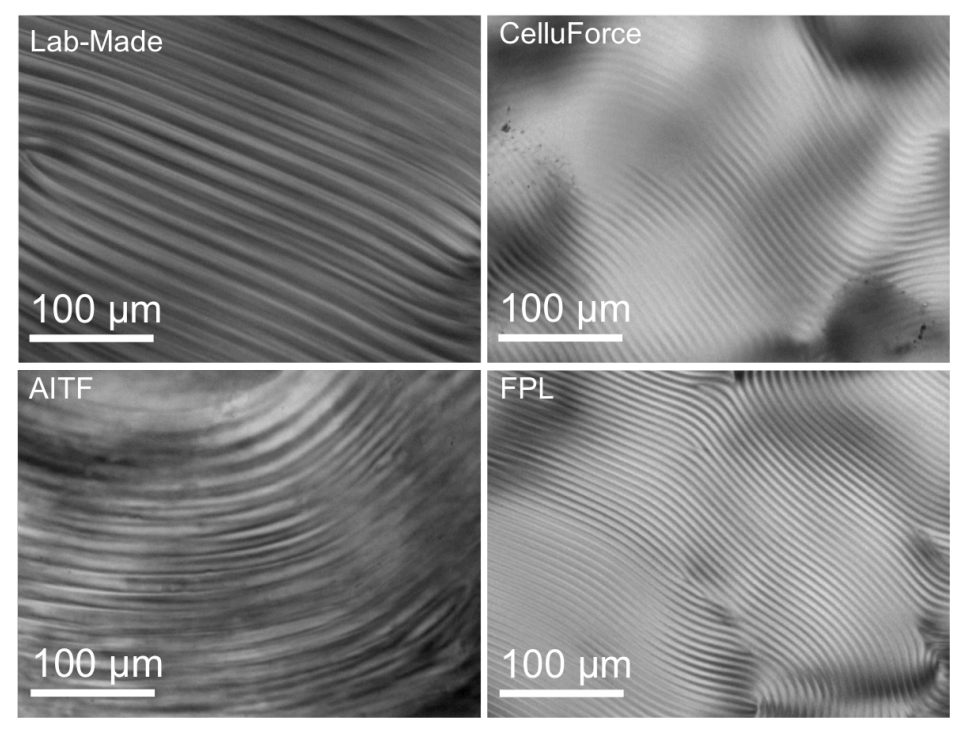

Figure 9: POM images of the anisotropic phase of "as received" CNCs, showing clear fingerprint texture in all samples indicating chiral nematic liquid crystal organization. Concentrations are: Lab-Made $8 \mathrm{wt} \%$, CelluForce $3.5 \mathrm{wt} \%$, AITF $8 \mathrm{wt} \%$, and FPL $7 \mathrm{wt} \%$. 


\section{Effect of Changing Cellulose Source and Production Scale}

AITF CNCs investigated throughout this work were produced via bench scale conventional sulfuric acid hydrolysis of cotton. More recently, AITF has begun to produce CNCs at the pilot scale using wood sourced cellulose. Table 4 shows the properties of both cotton and softwood dissolving pulp-derived CNCs in which particles have identical crystallinity (see Supporting Information Figure S9), and sulfate half ester content. Small changes in the zeta potential apparent size by DLS and thermal stability (see Supporting Information Figure S8) are evident, but expected when transitioning to wood cellulose starting materials. Additionally Figure 10 shows phase separation and self-assembly properties are maintained.

Table 4: Properties of AITF "as received" CNCs derived from cotton and softwood dissolving pulp.

\begin{tabular}{lcc} 
& \multicolumn{2}{c}{ Cellulose Source } \\
\cline { 2 - 3 } & Cotton & Softwood Dissolving Pulp \\
\hline \hline gS/100g CNC & $0.69 \pm 0.03$ & $0.69 \pm 0.02$ \\
R-OSO ${ }_{3} \mathrm{H} \mathrm{mmol} / \mathrm{kg} \mathrm{CNC}$ & $215 \pm 10$ & $215 \pm 5$ \\
Zeta Potential (mV) & $-42 \pm 1$ & $-34 \pm 1$ \\
Apparent Size by DLS (nm) & $73 \pm 2$ & $88 \pm 16$ \\
Crystallinity (\%) & 91 & 91
\end{tabular}



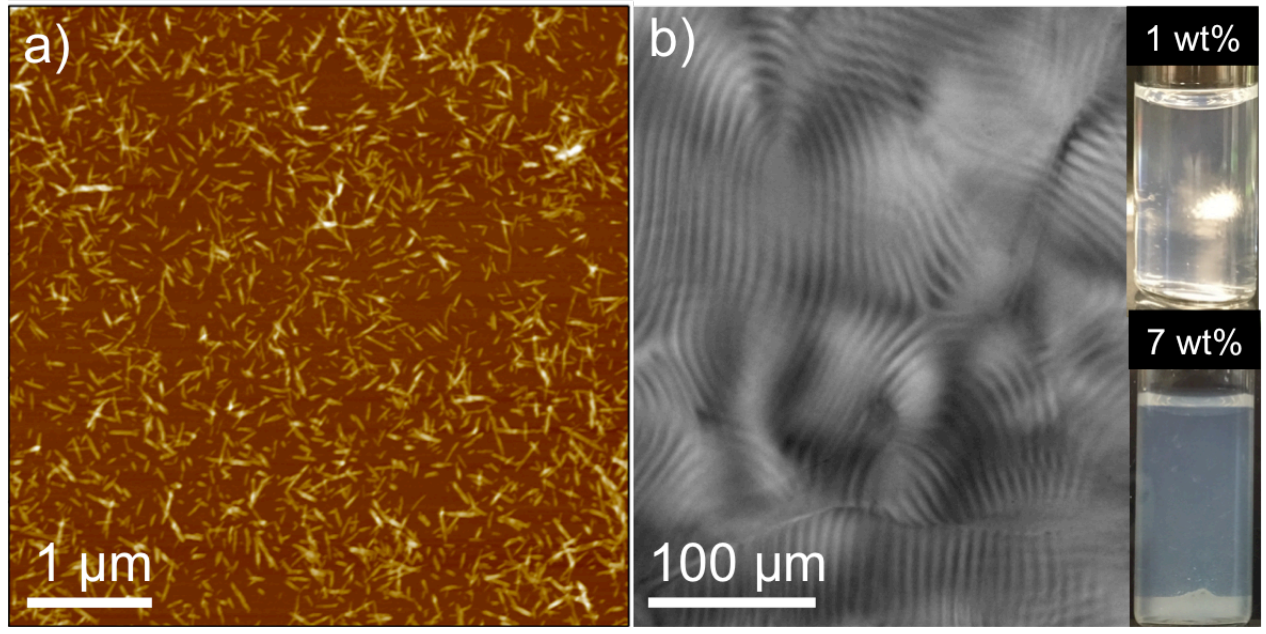

Figure 10: (a) AFM height image of AITF "as received" CNCs from softwood dissolving pulp.

(b) POM image of anisotropic phase with visual appearance of dilute and concentrated CNC suspensions.

\section{Other Nanocelluloses Produced through Non-Conventional Methods}

In addition to the traditional sulfuric acid hydrolysis, several industrial producers are now extracting $\mathrm{CNCs} / \mathrm{CNFs}$ using less conventional methods and there has yet to be any significant comparison with sulfuric acid extracted CNCs. Importantly, some of these processes do not use acid hydrolysis, and as a result no electrostatic stabilization can be expected.

American Process Inc.

Figure 11 shows an optical microscopy image of BioPlus ${ }^{\mathrm{TM}}$ Crystals along with the appearance of a $1 \mathrm{wt} \%$ dispersion. Clearly a significant portion of the material contains macroscopic fibers leading to highly turbid dispersions. The crystallinity was measured by XRD to be $>85 \%$ (see Supporting Information Figure S10) and AFM imaging (Figure 11 inset) shows the presence of both microscopic and nanoscale cellulose material. Importantly because the extraction process does not graft charged groups to the nanoparticle surface, API CNCs demonstrated poor colloidal 
stability and settled after approximately 1 hour - as such, no reliable DLS or zeta potential measurements could be obtained.

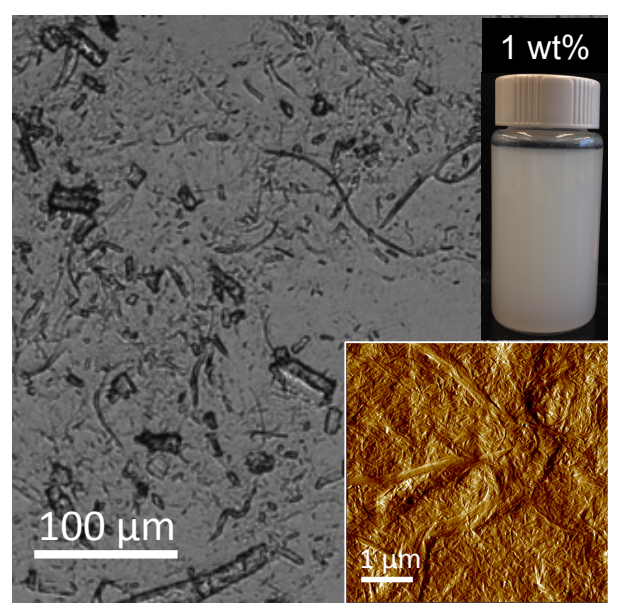

Figure 11: Optical microscopy image showing macroscopic fibers with BioPlus ${ }^{\mathrm{TM}}$ Crystals along with a photograph of a $1 \mathrm{wt} \%$ dispersion. Inset shows $5 \mu \mathrm{m} \times 5 \mu \mathrm{m}$ AFM amplitude image in which nanocellulose structures are visible.

\section{Blue Goose Biorefineries}

Blue Goose Biorefineries uses a proprietary transition metal catalyzed oxidative process to extract highly crystalline, $>90 \%$ (see Supporting Information, Figure S11), carboxyl functionalized nanocellulose material. Figure 12 shows $1 \mathrm{wt} \%$ dispersion of BGB Natural ${ }^{\mathrm{TM}}$ along with AFM images of "as received" material showing high aspect ratio nanofibrils with some microscopic components. No macroscopic fibers were evident by eye or optical microscopy. DLS measurements exhibit a bimodal size distribution with peaks centered at $40-$ $50 \mathrm{~nm}$ and 235 - $272 \mathrm{~nm}$ (see Supporting Information Figure S12) in good agreement with AFM images. BGB Natural ${ }^{\mathrm{TM}}$ particles show good colloidal stability with zeta potential of $-18 \pm 1 \mathrm{mV}$. 


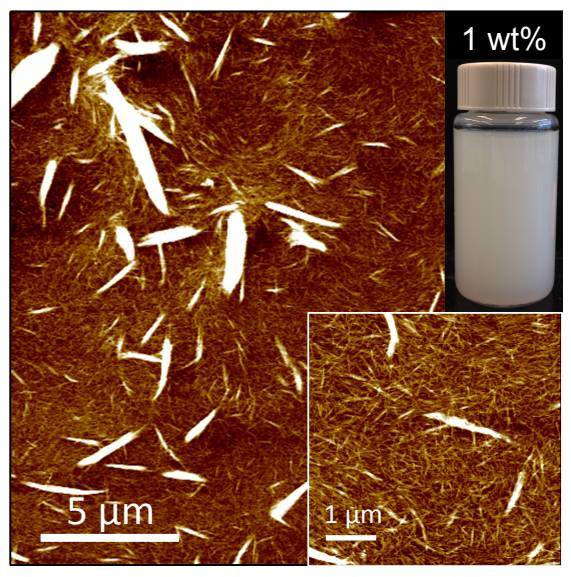

Figure 12: AFM height image of BGB Natural ${ }^{\mathrm{TM}}$ showing microscopic material and a $1 \mathrm{wt} \%$ dispersion. Inset shows $5 \mu \mathrm{m} \times 5 \mu \mathrm{m}$ AFM height image.

\section{Discussion}

CNC Properties from Different Producers. Overall, the sulfuric acid hydrolyzed CNCs produced industrially compare well with conventional lab made CNCs. In all cases sulfuric acid hydrolysis produced high aspect ratio particles that were electrostatically stabilized via grafted sulfate half ester groups. Moreover, once producers have a good "feel" for CNC production at the bench scale it seems that scale-up and even changes in starting cellulose material have minimal impact on the $\mathrm{CNC}$ chemical and physical properties. Although the CNCs investigated had similar properties, we emphasize that slight variations in these properties can have a significant effect on $\mathrm{CNC}$ behaviour and thorough characterization is still recommended prior to use. For example, using CNCs as rheological modifiers, reinforcing agents, or emulsion/foam stabilizers, could be severely impacted such that reproducing published results with CNCs of varying properties (e.g. CNCs from different manufacturers) would present significant challenges. Below we discuss key property differences of "as received" materials and the potential impact in various applications. 
Sulfate half ester content is a key factor in CNC behaviour and is responsible for the colloidal stability of the particles. Notably the sulfate half ester content of FPL CNCs was nearly $25 \%$ greater than other producers (Figure 2). Interestingly this trend is not reflected in the zeta potential or thermal stability as FPL and Lab-Made CNCs are equivalent. Critically, electrophoretic mobility, and ultimately zeta potential, is influenced not only by sulfate ester content but particle shape and surface morphology. Zeta potential measurements indicate that even though FPL CNCs have a higher sulfate half ester content their mobility in an electric field is similar to other CNCs tested. Although, higher charge density potentially imparts superior colloidal stability, particularly in environments with elevated ionic strengths, it has been shown to have a dramatic effect on how particles behave at interfaces. Kalashnikova et al. demonstrated that charge density plays a major role in how effective CNCs are as Pickering emulsion stabilizers leaning towards lower charged CNCs for more stable emulsions. ${ }^{117}$

$\mathrm{CNC}$ behaviour at an interface is not only dependent on surface charge density but particle aspect ratio as well. In a follow-up publication Kalashnikova et al. further demonstrated that the coverage and behaviour of oil-in-water emulsions was heavily influenced by particle aspect ratio. ${ }^{75}$ Using shorter particles as stabilizing agents produced more individualized droplets where as long CNCs bridged droplets creating network-like emulsions. Aspect ratio not only affects interfacial behaviour but impacts, rheological properties, ${ }^{73}$ reinforcing capability, ${ }^{76}$ and packing of CNCs. ${ }^{118}$ Particles with higher aspect ratios, form gels and percolated networks at significantly lower concentrations than shorter particles, potentially reducing the amount of material required for specific applications. Again this highlights that thorough characterization is needed since cellulose source and extraction procedures impact the aspect ratio as evidenced by CelluForce CNCs, which have a significantly larger aspect ratio. 
We emphasize that microscopy is an essential tool for characterization since DLS measurements, although giving relative particle sizes and distributions, do not convey true particle shape, dimensions, and size distribution. Clear evidence of this is seen when analyzing the dimensions of FPL CNCs as dispersions and in the dry state. DLS measurements suggest that FPL CNCs are more than $10 \mathrm{~nm}$ smaller than other CNCs (Figure 4) however AFM analysis of both the length and cross section show FPL CNCs are equivalent to other producers. However, DLS does suggest that FPL CNCs have a higher diffusion constant, which is perhaps a result of cellulose II precipitating on the surface and reducing the "fuzziness" that is sometimes attributed to oligosaccharides on the $\mathrm{CNC}$ surface. ${ }^{72}$

A critical feature of CNCs is the cellulose I crystal structure. Cellulose I is known not to swell and thus CNCs are expected to maintain their size and morphology in a variety of solvents and matrices. ${ }^{101}$ Additionally, the high elastic modulus and potential reinforcing capabilities of CNCs is largely attributed to the rigid cellulose I crystal structure and thus, highly crystalline materials are desired for these applications. CNCs produced by Lab-Made, CelluForce and AITF are highly crystalline with cellulose I percentages upwards of ca. $90 \%$ in good agreement with previous literature values. Most notable in the crystallinity measurements is the presence of cellulose II within FPL CNCs which has been observed previously. ${ }^{107}$ Although we can only postulate as to the source of cellulose II we suggest that a portion of the cellulose I structure was at least partially dissolved in $\mathrm{NaOH}$ and subsequently re-generated/precipitated onto the $\mathrm{CNC}$ surface into the cellulose II polymorph. We note the dissolving pulp used in the production of FPL CNC as a possible source of the cellulose II and they suggest that $\mathrm{NaOH}$ concentrations used in CNC production are below the threshold for mercerization. Currently, presence of 
cellulose II with FPL material appears to have little impact on the particle behaviour, as it maintains good colloidal stability, aspect ratio and self-assembly.

Both industrially produced and bench scale CNCs show good thermal stability up to $250^{\circ} \mathrm{C}$, suggesting that incorporating CNCs into drilling fluids and polymer composites via melt mixing is feasible for all as received CNCs. Moreover, while sulfate half ester groups do fall off with heating and over time ${ }^{90}$ this work implies that the amount of sulfate esters is not directly linked to $\mathrm{CNC}$ thermal stability within the range investigated. Although not tested in this work, we emphasize that the rate of heating (i.e. incorporating directly into a polymer melt vs. a gradual temperature increase) along with the bound moisture content is related to discoloration and remains poorly understood.

Biphasic behaviour and more specifically the formation of anisotropic chiral nematic structures above a critical concentration is an emblem of good $\mathrm{CNC}$ behaviour and is of great interest as structured colour templates for cosmetics, anti-counterfeit and optical applications. ${ }^{119}$ The formation of these structures in all of the CNCs investigated is an advantage for future applications. However, despite the many academic demonstrations and control of CNC selfassembly in magnetic ${ }^{34-39}$ and electric fields, ${ }^{40-42}$ exploitation of this behaviour in a commercially relevant and scalable processes remains to be seen.

Soxhlet extraction. Generally Soxhlet extraction had a minimal effect on CNC properties. Only minor changes in the sulfate half ester content, zeta potential and apparent particle size were observed for all CNCs. Where these values differ it is believed that $\mathrm{CNC}$ behaviour, such as colloidal stability, still remain relatively unchanged. For example, the zeta potential of Lab-Made and FPL CNCs increased by ca. $-5 \mathrm{mV}$ following Soxhlet extraction, however particles remain 
highly anionic and stable with zeta potentials of ca. $-40 \mathrm{mV}$. This trend is similarly reflected in the sulfate ester content where the largest decrease of $7.5 \%$ was observed for FPL CNCs. The apparent particle diameter as measured by DLS indicates that the particle dimensions are largely unchanged following Soxhlet extraction and any increase in size is attributed to aggregation following exposure to ethanol.

While we believe that the bulk colloidal, thermal and interfacial behaviour of the CNCs will remain unchanged following Soxhlet extraction, this is not to say that Soxhlet extraction is not sometimes a pre-requisite. The recent publication by Bouchard et al., ${ }^{72}$ clearly demonstrates that bound oligosaccharides are very much impacted by the exact hydrolysis conditions. Some applications may need these and other byproducts removed to improve efficiency and reproducibility. Experiments which have demonstrated extreme sensitivity to purification protocols include grafting small molecules to $\mathrm{CNCs}$ (and polymer grafting ${ }^{79}$ ) and particularly when studying CNCs as model cellulose films - "open film" assembly on different substrates is strongly reliant on Soxlet extraction for repeatable experiments. ${ }^{120}$

The most significant impact of Soxhlet extraction observed in this work was in the crystal structure of FPL CNCs. Prior to extraction "as received" material had nearly a 1:1 ratio of cellulose I and cellulose II polymorphs. Following extraction, cellulose II content significantly decreased yielding a cellulose I to cellulose II ratio of 3:1 and suggesting that cellulose II is lost during purification. Additionally, due to the decrease in sulfate ester content and zeta potential, a portion of the cellulose II is presumably sulfated. We note however, that the loss of cellulose II does not significantly affect $\mathrm{CNC}$ behaviour as only minor changes in the apparent particle size and zeta potential were observed. 
X-Ray Photoelectron Spectroscopy. Soxhlet extraction is proposed to remove residual hydrolysis byproducts and oligomers from the $\mathrm{CNC}$ surface. Labet and Thielemans showed that following Soxhlet extraction the overall functionalization and reproducibility of ring opening polymerization onto CNCs was improved. ${ }^{79}$ Although, Soxhlet extraction improves functionalization, quantifying the purity of CNCs is challenging, particularly via XPS. Notably, any residual oligomers or hydrolysis byproducts that have precipitated onto the CNC surface will be primarily composed of cellulosic materials, chemically equivalent to CNCs. Moreover, clean cellulose filter paper has been shown to contain $5-7 \%$ aliphatic $\mathrm{C} 1$ carbon and that, storing samples in glass, aluminum plastic or cellophane can dramatically reduce reproducibility. ${ }^{110}$ This is particularly noteworthy for industrially produced $\mathrm{CNCs}$, since the various handling steps and shipping containers are unknown. As a result, we discourage the use of XPS for "baseline" CNC characterization but recognize its importance for examining nanocellulose modified with noncarbon/oxygen-only compounds.

Other Nanocelluloses. Nanocelluloses produced by American Process Inc. and Blue Goose Biorefineries, although highly crystalline with a significant "nano" component show important differences in appearance and properties compared to CNCs extracted by sulfuric acid hydrolysis. Most notable is the presence of macroscopic fibers in American Process Inc. BioPlus ${ }^{\mathrm{TM}}$ Crystals. These large-scale fibers will dramatically affect the rheological, colloidal and mechanical properties of the material, which could be positive for some applications (i.e. composites) but detrimental to others and ultimately should not be classified as CNCs. From our perspective, the work we have published on emulsions, hydrogels, aerogels, thin films and composites would not give the same results if API nanocellulose was used. Conversely, BGB Natural ${ }^{\mathrm{TM}}$ particles 
although containing a bimodal distribution of both nanocellulose and fibrillar cellulose showed no evidence of macroscopic material and demonstrated better colloidal stability with zeta potential of $-18 \pm 1 \mathrm{mV}$. We additionally note that Blue Goose Biorefineries reports that their more recent product, BGB Ultra ${ }^{\mathrm{TM}}$, although not investigated in this work, has a similar size distribution and optical properties to sulfuric acid extracted CNCs. The various products being produced from nanocellulose suppliers again highlights the need for thorough characterization prior to CNC use.

\section{Conclusion}

This work presents the first detailed analysis and comparison of laboratory and industrially produced sulfuric acid extracted CNCs and seeks to answer the questions:

\section{1) Are CNCs from various producers equivalent?}

Overall, industrially produced CNCs from CelluForce, Alberta Innovates Technology Futures and the USDA Forest Products Labs compare well with CNCs extracted at the bench scale with all material containing highly crystalline, high aspect ratio "nano-only" CNCs. However, differences in sulfate half ester content, colloidal stability, crystallinity and morphology do exist. These properties must be fully characterized prior to use as they can significantly impact particle behaviour.

Nanocelluloses produced by non-traditional processes investigated in this work, including American Process Inc. BioPlus ${ }^{\mathrm{TM}}$ Crystals and Blue Goose Biorefineries BGB Natural ${ }^{\mathrm{TM}}$, 
although containing highly crystalline nanocellulose material, have behaviour and properties that differ significantly from sulfuric acid extracted CNCs. Additionally the materials tested in this work cannot be considered in the same light as CNCs as they contain micro and macroscopic components.

2) What are the most practical characterization methods that should be employed prior to using CNCs?

This work has shown that changes to the cellulose source and hydrolysis conditions can significantly impact $\mathrm{CNC}$ properties and behaviour. As such, we emphasize that CNCs must be thoroughly characterized to ensure performance is consistent. For the majority of applications we recommend at minimum: conductometric titrations, zeta potential, XRD, dynamic light scattering, and microscopy (either AFM or TEM), to characterize the sulfate half ester content, colloidal stability, crystallinity and particle morphology. In applications in which CNCs are being surface modified, such as through polymer grafting, we further suggest including XPS before and after modification, however quantitative analysis remains difficult. We additionally note that as $\mathrm{CNC}$ applications increase in scale extensive characterization will become onerous and industrial scale quality assurance/control protocols must be developed.

3) Is further purification, such as Soxhlet extraction, required?

Our investigation shows that Soxhlet extraction does not significantly impact the "core" properties or behaviour of CNCs. Although minor changes were observed for the sulfate half 
ester content and zeta potential, particles remain highly negatively charged and colloidally stable. Moreover, quantifying the "cleanliness" remains extremely challenging. The most notable change following Soxhlet extract was observed in the crystallinity of FPL CNCs however particle physical properties did not change significantly.

4) If producers change scale and starting material, what kind of changes can we expect?

As cellulose sources and production scales change, obtaining identical material from industrial producers could be challenging and is worth watching out for. Moreover as $\mathrm{CNC}$ technology continues to develop we can expect a variety of nanocellulose grades from industrial producers with different properties and morphologies such as Blue Goose Biorefineries BGB Natural ${ }^{\mathrm{TM}}$ and BGB Ultra ${ }^{\mathrm{TM}}$. Currently, batch-to-batch variability will likely result in minor differences in particle dimensions and sulfate half ester content. Nonetheless, transitioning between cellulose sources and increasing production scale yielded comparable CNCs as evident from cotton and wood-based CNCs from AITF.

Ultimately for the $\mathrm{CNC}$ community to continue to grow, and for commercial applications to be realized, industrially produced $\mathrm{CNCs}$ are needed. Overall, this investigation demonstrates that with proper characterization, the transition from lab scale to industrial CNCs should be met with optimism. Current producers of sulfuric acid extracted CNCs are generating material with comparable particle dimensions and properties to those that have been investigated in the past. 


\section{Supporting Information}

X-ray diffraction patterns (Figures S1-S4 and S9-S11) and thermal gravimetric analysis profiles

(Figures S5-S8) of "as received" and Soxhlet extracted cellulose nanocrystals produced in the lab and at the industrial scale.

\section{Acknowledgements}

Funding from the Natural Sciences and Engineering Research Council of Canada, Industrial Postgraduate Scholarship program sponsored by Cabot Corporation is gratefully acknowledged. The authors also thank Richard Berry at CelluForce, Alan Rudie at the USDA Forest Products Lab, Behzad Ahvazi and Christophe Danumah at Alberta Innovates Technology Futures and Sean McAlpine at Blue Goose Biorefineries. Professors R. Pelton, A. Guarne and J. MoranMirabal are thanked for sharing equipment to characterize CNCs. We thank Victoria Jarvis as a part of the McMaster Analytical X-Ray Diffraction Facility for her time and expertise. Additionally, the Biointerfaces Institute and the Brockhouse Institute for Materials Research at McMaster University are acknowledged for support and equipment.

\section{References}

(1) Sionkowska, A. Current Research on the Blends of Natural and Synthetic Polymers as New Biomaterials: Review. Prog. Polym. Sci. 2011, 36 (9), 1254-1276.

(2) Yang, X.; Shi, K.; Zhitomirsky, I.; Cranston, E. D. Cellulose Nanocrystal Aerogels as Universal 3D Lightweight Substrates for Supercapacitor Materials. Adv. Mater. 2015, 27 (40), 6104-6109.

(3) Nyström, G.; Marais, A.; Karabulut, E.; Wågberg, L.; Cui, Y.; Hamedi, M. M. Self-Assembled Three-Dimensional and Compressible Interdigitated Thin-Film Supercapacitors and Batteries. Nat. Commun. 2015, 6 (May), 7259. 
(4) Nyström, G.; Fernández-Ronco, M. P.; Bolisetty, S.; Mazzotti, M.; Mezzenga, R. Amyloid Templated Gold Aerogels. Adv. Mater. 2016, 28 (3), 472-478.

(5) Xie, F.; Halley, P. J.; Avérous, L. Rheology to Understand and Optimize Processibility, Structures and Properties of Starch Polymeric Materials. Prog. Polym. Sci. 2012, 37 (4), 595-623.

(6) Yang, X.; Bakaic, E.; Hoare, T.; Cranston, E. D. Injectable Polysaccharide Hydrogels Reinforced with Cellulose Nanocrystals: Morphology, Rheology, Degradation, and Cytotoxicity. Biomacromolecules 2013, 14 (12), 4447-4455.

(7) La Mantia, F. P.; Morreale, M. Green Composites: A Brief Review. Compos. Part A Appl. Sci. Manuf. 2011, 42 (6), 579-588.

(8) Siqueira, G.; Bras, J.; Dufresne, A. Cellulosic Bionanocomposites: A Review of Preparation, Properties and Applications. Polymers (Basel). 2010, 2 (4), 728-765.

(9) Eichhorn, S. J.; Baillie, C. A.; Zafeiropoulos, N.; Mwaikambo, L. Y.; Ansell, M. P.; Dufresne, A.; Entwistle, K. M.; Herrera-Franco, P. J.; Escamilla, G. C.; Groom, L.; et al. Current International Research into Cellulosic Fibres and Composites. J. Mater. Sci. 2001, 36, 2107-2131.

(10) Faruk, O.; Bledzki, A. K.; Fink, H. P.; Sain, M. Biocomposites Reinforced with Natural Fibers: 2000-2010. Prog. Polym. Sci. 2012, 37 (11), 1552-1596.

(11) Liu, X.; Wang, Y.; Yu, L.; Tong, Z.; Chen, L. Thermal Degradation and Stability of Starch under Different Processing Conditions. Starch-Stärke 2013, 48-60.

(12) Liu, H.; Xie, F.; Yu, L.; Chen, L.; Li, L. Thermal Processing of Starch-Based Polymers. Prog. Polym. Sci. 2009, 34 (12), 1348-1368.

(13) Pillai, C. K. S.; Paul, W.; Sharma, C. P. Chitin and Chitosan Polymers: Chemistry, Solubility and Fiber Formation. Prog. Polym. Sci. 2009, 34 (7), 641-678.

(14) Rinaudo, M.; Ã, M. R. Chitin and Chitosan: Properties and Applications. Prog. Polym. Sci. 2006, $31(7), 603-632$.

(15) Gallezot, P. Conversion of Biomass to Selected Chemical Products. Chem. Soc. Rev. 2012, 41 (4), $1538-1558$.

(16) Klemm, D.; Heublein, B.; Fink, H. P.; Bohn, A. Cellulose: Fascinating Biopolymer and Sustainable Raw Material. Angew. Chemie - Int. Ed. 2005, 44 (22), 3358-3393.

(17) Schaefer, D. W.; Justice, R. S. How Nano Are Nanocomposites? Macromolecules 2007, 40 (24), 8501-8517.

(18) Klemm, D.; Kramer, F.; Moritz, S.; Lindström, T.; Ankerfors, M.; Gray, D. G.; Dorris, A. Nanocelluloses: A New Family of Nature-Based Materials. Angew. Chemie - Int. Ed. 2011, 50, $5438-5466$. 
(19) Nickerson, R. F.; Habrle, J. a. Cellulose Intercrystalline Structure. Ind. Eng. Chem. 1947, 39 (11), $1507-1512$.

(20) Ranby, B. .; Ribi, E. Uber Den Feinbau Zellulose. Experientia 1950, 6, 12-14.

(21) Brinchi, L.; Cotana, F.; Fortunati, E.; Kenny, J. M. Production of Nanocrystalline Cellulose from Lignocellulosic Biomass: Technology and Applications. Carbohydr. Polym. 2013, 94 (1), 154 169.

(22) Medronho, B.; Romano, A.; Miguel, M. G.; Stigsson, L.; Lindman, B. Rationalizing Cellulose (in)solubility: Reviewing Basic Physicochemical Aspects and Role of Hydrophobic Interactions. Cellulose 2012, 19, 581-587.

(23) Moon, R. J.; Martini, A.; Nairn, J.; Simonsen, J.; Youngblood, J. Cellulose Nanomaterials Review: Structure, Properties and Nanocomposites. Chem. Soc. Rev. 2011, 40 (7), 3941-3994.

(24) Azizi Samir, M. A. S.; Alloin, F.; Dufresne, A. Review of Recent Research into Cellulosic Whiskers, Their Properties and Their Application in Nanocomposite Field. Biomacromolecules 2005, $6(2), 612-626$.

(25) Eichhorn, S. J.; Dufresne, A.; Aranguren, M.; Marcovich, N. E.; Capadona, J. R.; Rowan, S. J.; Weder, C.; Thielemans, W.; Roman, M.; Renneckar, S.; et al. Review: Current International Research into Cellulose Nanofibres and Nanocomposites. J. Mater. Sci. 2009, 45 (1), 1-33.

(26) Habibi, Y.; Lucia, L. A.; Rojas, O. J. Cellulose Nanocrystals: Chemistry, Self-Assembly, and Applications. Chem. Rev. 2010, 110 (6), 3479-3500.

(27) Habibi, Y. Key Advances in the Chemical Modification of Nanocelluloses. Chem. Soc. Rev. 2014, 43 (5), 1519-1542.

(28) Mariano, M.; El Kissi, N.; Dufresne, A. Cellulose Nanocrystals and Related Nanocomposites: Review of Some Properties and Challenges. J. Polym. Sci. Part B Polym. Phys. 2014, 52, 791806.

(29) Zhu, H.; Luo, W.; Ciesielski, P. N.; Fang, Z.; Zhu, J. Y.; Henriksson, G.; Himmel, M. E.; Hu, L. Wood-Derived Materials for Green Electronics, Biological Devices, and Energy Applications. Chem. Rev. 2016, 116 (16), 9305-9374.

(30) Revol, J. F.; Bradford, H.; Giasson, J.; Marchessault, R. H.; Gray, D. G. Helicoidal Self-Ordering of Cellulose Microfibrils in Aqueous Suspension. Int. J. Biol. Macromol. 1992, 14 (3), 170-172.

(31) Dong, X. M.; Kimura, T.; Revol, J.-F.; Gray, D. G. Effects of Ionic Strength on the Isotropic-Chiral Nematic Phase Transition of Suspensions of Cellulose Crystallites. Langmuir 1996, 12 (8), 2076-2082.

(32) Dong, X. M.; Gray, D. G. Effect of Counterions on Ordered Phase Formation in Suspensions of Charged Rodlike Cellulose Crystallites. Langmuir 1997, 13 (8), 2404-2409. 
(33) Lagerwall, J. P. F.; Schütz, C.; Salajkova, M.; Noh, J.; Hyun Park, J.; Scalia, G.; Bergström, L. Cellulose Nanocrystal-Based Materials: From Liquid Crystal Self-Assembly and Glass Formation to Multifunctional Thin Films. NPG Asia Mater. 2014, 6 (1), e80.

(34) De France, K. J.; Yager, K. G.; Hoare, T.; Cranston, E. D. Cooperative Ordering and Kinetics of Cellulose Nanocrystal Alignment in a Magnetic Field. Langmuir 2016, 32 (30), 7564-7571.

(35) Frka-Petesic, B.; Sugiyama, J.; Kimura, S.; Chanzy, H.; Maret, G. Negative Diamagnetic Anisotropy and Birefringence of Cellulose Nanocrystals. Macromolecules 2015, 48 (24), 8844 8857.

(36) Pullawan, T.; Wilkinson, A. N.; Eichhorn, S. J. Influence of Magnetic Field Alignment of Cellulose Whiskers on the Mechanics of All-Cellulose Nanocomposites. Biomacromolecules 2012, 13 (8), 2528-2536.

(37) Revol, J.-F.; Godbout, L.; Dong, X.-M.; Gray, D. G.; Chanzy, H.; Maret, G. Chiral Nematic Suspensions of Cellulose Crystallites; Phase Separation and Magnetic Field Orientation. Liq. Cryst. 1994, 16 (1), 127-134.

(38) Song, G.; Kimura, F.; Kimura, T.; Piao, G. Orientational Distribution of Cellulose Nanocrystals in a Cellulose Whisker as Studied by Diamagnetic Anisotropy. Macromolecules 2013, 46 (22), 8957-8963.

(39) Kim, D. H.; Song, Y. S. Rheological Behavior of Cellulose Nanowhisker Suspension under Magnetic Field. Carbohydr. Polym. 2015, 126, 240-247.

(40) Csoka, L.; Hoeger, I. C.; Peralta, P.; Peszlen, I.; Rojas, O. J. Dielectrophoresis of Cellulose Nanocrystals and Alignment in Ultrathin Films by Electric Field-Assisted Shear Assembly. $J$. Colloid Interface Sci. 2011, 363 (1), 206-212.

(41) Frka-Petesic, B.; Jean, B.; Heux, L. First Experimental Evidence of a Giant Permanent ElectricDipole Moment in Cellulose Nanocrystals. EPL (Europhysics Lett. 2014, 107 (2), 28006.

(42) Bordel, D.; Putaux, J.-L.; Heux, L. Orientation of Native Cellulose in an Electric Field. Langmuir 2006, 22 (11), 4899-4901.

(43) Csoka, L.; Hoeger, I. C.; Rojas, O. J.; Peszlen, I.; Pawlak, J. J.; Peralta, P. N. Piezoelectric Effect of Cellulose Nanocrystals Thin Films. ACS Macro Lett. 2012, 1 (7), 867-870.

(44) Hu, Z.; Patten, T.; Pelton, R.; Cranston, E. D. Synergistic Stabilization of Emulsions and Emulsion Gels with Water-Soluble Polymers and Cellulose Nanocrystals. ACS Sustain. Chem. Eng. 2015, 3 (5), 1023-1031.

(45) Hu, Z.; Cranston, E. D.; Ng, R.; Pelton, R. Tuning Cellulose Nanocrystal Gelation with Polysaccharides and Surfactants. Langmuir 2014, 30 (10), 2684-2692.

(46) Hu, Z.; Ballinger, S.; Pelton, R.; Cranston, E. D. Surfactant-Enhanced Cellulose Nanocrystal Pickering Emulsions. J. Colloid Interface Sci. 2014, 439, 139-148. 
(47) Hu, Z.; Marway, H. S.; Kasem, H.; Pelton, R.; Cranston, E. D. Dried and Redispersible Cellulose Nanocrystal Pickering Emulsions. ACS Macro Lett. 2015, 5, 185-789.

(48) Chau, M.; De France, K. J.; Kopera, B.; Machado, V. R.; Rosenfeldt, S.; Reyes, L.; Chan, K. J. W.; Förster, S.; Cranston, E. D.; Hoare, T.; et al. Composite Hydrogels with Tunable Anisotropic Morphologies and Mechanical Properties. Chem. Mater. 2016, 28 (10), 3406-3415.

(49) Hu, Z.; Xu, R.; Cranston, E. D.; Pelton, R. Stable Aqueous Foams from Cellulose Nanocrystals and Methyl Cellulose. Biomacromolecules 2016, submitted.

(50) De France, K. J.; Chan, K. J. W.; Cranston, E. D.; Hoare, T. Enhanced Mechanical Properties in Cellulose Nanocrystal-Poly(oligoethylene Glycol Methacrylate) Injectable Nanocomposite Hydrogels through Control of Physical and Chemical Cross-Linking. Biomacromolecules 2016, 17 (2), 649-660.

(51) Kedzior, S. a.; Graham, L.; Moorlag, C.; Dooley, B. M.; Cranston, E. D. Poly(methyl Methacrylate)-Grafted Cellulose Nanocrystals: One-Step Synthesis, Nanocomposite Preparation, and Characterization. Can. J. Chem. Eng. 2016, 94 (5), 811-822.

(52) Kan, K. H. M.; Li, J.; Wijesekera, K.; Cranston, E. D. Polymer-Grafted Cellulose Nanocrystals as pH-Responsive Reversible Flocculants. Biomacromolecules 2013, 14 (9), 3130-3139.

(53) Rivkin, A.; Abitbol, T.; Nevo, Y.; Verker, R.; Lapidot, S.; Komarov, A.; Veldhuis, S. C.; Zilberman, G.; Reches, M.; Cranston, E. D.; et al. Bionanocomposite Films from Resilin-CBD Bound to Cellulose Nanocrystals. Ind. Biotechnol. 2015, 11, 44-58.

(54) Yang, X.; Cranston, E. D. Chemically Cross-Linked Cellulose Nanocrystal Aerogels with Shape Recovery and Superabsorbent Properties. Chem. Mater. 2014, 26 (2), 6016-6025.

(55) Abitbol, T.; Marway, H.; Cranston, E. D. Surface Modification of Cellulose Nanocrystals with Cetyltrimethylammonium Bromide. Nord. Pulp Pap. Res. J. 2014, 29 (1), 46-57.

(56) Cranston, E. D.; Gray, D. G. Morphological and Optical Characterization of Polyelectrolyte Multilayers Incorporating Nanocrystalline Cellulose. Biomacromolecules 2006, 7 (9), 2522-2530.

(57) Villalpando-Páez, F.; Nguyen, L.; Abitbol, T.; Komarov, A.; Prevo, B. G.; Corwin, J.; Galli, C.; Choudhary, S.; Veldhuis, S. C.; Villalobos, M.; et al. Comparison of Nanocrystalline Cellulose and Fumed Silica in Latex Coatings. Green Mater. 2014, 2, 206-221.

(58) Shi, K.; Yang, X.; Cranston, E. D.; Zhitomirsky, I. Efficient Lightweight Supercapacitor with Compression Stability. Adv. Funct. Mater. 2016, 26 (35), 6437-6445.

(59) Zhu, H.; Yang, X.; Cranston, E. D.; Zhu, S. Flexible and Porous Nanocellulose Aerogels with High Loadings of Metal-Organic Framework Particles for Separations Applications. Adv. Mater. 2016, 28 (35), 7652-7765.

(60) Gill, U.; Sutherland, T.; Himbert, S.; Rheinstädter, M.; Cranston, E. D.; Moran-Mirabal, J. M. Beyond Buckling: Humidity-Independent Measurement of the Mechanical Properties of Green Nanocomposite Films. Small 2016, submitted. 
(61) Cranston, E. D.; Gray, D. G.; Rutland, M. W. Direct Surface Force Measurements of Polyelectrolyte Multilayer Films Containing Nanocrystalline Cellulose. Langmuir 2010, 26 (22), 17190-17197.

(62) Sato, T.; Ali, M. M.; Pelton, R.; Cranston, E. D. DNA Stickers Promote Polymer Adsorption onto Cellulose. Biomacromolecules 2012, 13 (10), 3173-3180.

(63) Reid, M. S.; Villalobos, M.; Cranston, E. D. Cellulose Nanocrystal Interactions Probed by Thin Film Swelling to Predict Dispersibility. Nanoscale 2016, 8 (24), 12247-12257.

(64) Abitbol, T.; Palermo, A.; Moran-Mirabal, J. M.; Cranston, E. D. Fluorescent Labeling and Characterization of Cellulose Nanocrystals with Varying Charge Contents. Biomacromolecules 2013, 14 (9), 3278-3284.

(65) Sacui, I. a.; Nieuwendaal, R. C.; Burnett, D. J.; Stranick, S. J.; Jorfi, M.; Weder, C.; Foster, E. J.; Olsson, R. T.; Gilman, J. W. Comparison of the Properties of Cellulose Nanocrystals and Cellulose Nanofibrils Isolated from Bacteria, Tunicate, and Wood Processed Using Acid, Enzymatic, Mechanical, and Oxidative Methods. ACS Appl. Mater. Interfaces 2014, 6 (9), 61276138.

(66) Hamad, W. Y.; Hu, T. Q. Structure-Process-Yield Interrelations in Nanocrystalline Cellulose Extraction. Can. J. Chem. Eng. 2010, 88 (3), 392-402.

(67) Chen, L.; Wang, Q.; Hirth, K.; Baez, C.; Agarwal, U. P.; Zhu, J. Y. Tailoring the Yield and Characteristics of Wood Cellulose Nanocrystals (CNC) Using Concentrated Acid Hydrolysis. Cellulose 2015, 22 (3), 1753-1762.

(68) Dong, X. M.; Revol, J.-F.; Gray, D. G. Effect of Microscystallite Preparation Conditions on the Formation of Colloid Crystals of Cellulose. Cellulose 1998, 5 (1), 19-32.

(69) Beck-Candanedo, S.; Roman, M.; Gray, D. G. Effect of Reaction Conditions on the Properties and Behavior of Wood Cellulose Nanocrystal Suspensions. Biomacromolecules 2005, 6 (2), 10481054.

(70) Dong, S.; Bortner, M. J.; Roman, M. Analysis of the Sulfuric Acid Hydrolysis of Wood Pulp for Cellulose Nanocrystal Production: A Central Composite Design Study. Ind. Crops Prod. 2016, 93, $76-87$.

(71) Wang, Q.; Zhao, X.; Zhu, J. Y. Kinetics of Strong Acid Hydrolysis of a Bleached Kraft Pulp for Producing Cellulose Nanocrystals (CNCs). Ind. Eng. Chem. Res. 2014, 53 (27), 11007-11014.

(72) Bouchard, J.; Méthot, M.; Fraschini, C.; Beck, S. Effect of Oligosaccharide Deposition on the Surface of Cellulose Nanocrystals as a Function of Acid Hydrolysis Temperature. Cellulose 2016.

(73) Wu, Q.; Meng, Y.; Wang, S.; Li, Y.; Fu, S.; Ma, L.; Harper, D. Rheological Behavior of Cellulose Nanocrystal Suspension: Influence of Concentration and Aspect Ratio. J. Appl. Polym. Sci. 2014, 131 (15), 40525-40533. 
(74) Cherhal, F.; Cousin, F.; Capron, I. Influence of Charge Density and Ionic Strength on the Aggregation Process of Cellulose Nanocrystals in Aqueous Suspension, as Revealed by SmallAngle Neutron Scattering. Langmuir 2015, 31 (20), 5596-5602.

(75) Kalashnikova, I.; Bizot, H.; Bertoncini, P.; Cathala, B.; Capron, I. Cellulosic Nanorods of Various Aspect Ratios for Oil in Water Pickering Emulsions. Soft Matter 2013, 9 (3), 952.

(76) Rusli, R.; Shanmuganathan, K.; Rowan, S. J.; Weder, C.; Eichhorn, S. J. Stress Transfer in Cellulose Nanowhisker Composites-Influence of Whisker Aspect Ratio and Surface Charge. Biomacromolecules 2011, 12 (4), 1363-1369.

(77) Beck, S.; Bouchard, J.; Berry, R. Controlling the Reflection Wavelength of Iridescent Solid Films of Nanocrystalline Cellulose. Biomacromolecules 2011, 12, 167-172.

(78) Shafiei-Sabet, S.; Hamad, W. Y.; Hatzikiriakos, S. G. Rheology of Nanocrystalline Cellulose Aqueous Suspensions. Langmuir 2012, 28 (49), 17124-17133.

(79) Labet, M.; Thielemans, W. Improving the Reproducibility of Chemical Reactions on the Surface of Cellulose Nanocrystals: ROP of E-Caprolactone as a Case Study. Cellulose 2011, 18 (3), $607-$ 617.

(80) Akpinar, O.; McGorrin, R. J.; Penner, M. H. Cellulose-Based Chromatography for Cellooligosaccharide Production. J. Agric. Food Chem. 2004, 52 (13), 4144-4148.

(81) Peri, S.; Muthukumar, L.; Nazmul Karim, M.; Khare, R. Dynamics of Cello-Oligosaccharides on a Cellulose Crystal Surface. Cellulose 2012, 19 (6), 1791-1806.

(82) Future Markets Inc. The Global Market for Nanocellulose to 2020; 2013.

(83) Microfibrillar Cellulose Project from Borregaard Gets EU Funding. BioPlastics Magazine. 2016.

(84) Canadian Standards Association. Cellulosic Nanomaterials - Test Methods for Characterization (CSA Z5100-14); 2014.

(85) Sarma, S. J.; Ayadi, M.; Brar, S. K.; Berry, R. Sustainable Commercial Nanocrystalline Cellulose Manufacturing Process with Acid Recycling. Carbohydr. Polym. 2017, 156, $26-33$.

(86) Houtman, C. Technoeconomic Analysis of Commercial-Scale CNC Production. In TAPPI Nano; 2016.

(87) Nelson, K.; Retsina, T. Innovative Nanocellulose Process Breaks the Cost Barrier. TAPPI J. 2014, $13(5), 19-23$.

(88) Nelson, K.; Retsina, T.; Iakovlev, M.; van Heiningen, A.; Deng, Y.; Shatkin, J. A.; Mulyadi, A. Materials Research for Manufacturing; 2016; Vol. 224.

Blue Goose Biorefineries Inc. http://bluegoosebiorefineries.com/ (accessed Sep 1, 2016). 
(90) Beck, S.; Bouchard, J. Auto-Catalyzed Acidic Desulfation of Cellulose Nanocrystals. Nord. Pulp Pap. Res. J. 2014, 29 (01), 006-014.

(91) Abitbol, T.; Kloser, E.; Gray, D. G. Estimation of the Surface Sulfur Content of Cellulose Nanocrystals Prepared by Sulfuric Acid Hydrolysis. Cellulose 2013, 20 (2), 785-794.

(92) Beck, S.; Méthot, M.; Bouchard, J. General Procedure for Determining Cellulose Nanocrystal Sulfate Half-Ester Content by Conductometric Titration. Cellulose 2014, 22 (1), 101-116.

(93) Agarwal, U. P.; Ralph, S. a.; Reiner, R. S.; Baez, C. Probing Crystallinity of Never-Dried Wood Cellulose with Raman Spectroscopy. Cellulose 2016, 23 (1), 125-144.

(94) Nishiyama, Y.; Langan, P.; Chanzy, H. Crystal Structure and Hydrogen-Bonding System in Cellulose Ibeta from Synchrotron X-Ray and Neutron Fiber Diffraction. J. Am. Chem. Soc. 2002, 124 (31), 9074-9082.

(95) Park, S.; Baker, J. O.; Himmel, M. E.; Parilla, P. a; Johnson, D. K. Cellulose Crystallinity Index: Measurement Techniques and Their Impact on Interpreting Cellulase Performance. Biotechnol. Biofuels 2010, 3 (1), 10.

(96) French, A. D.; Santiago Cintrón, M. Cellulose Polymorphy, Crystallite Size, and the Segal Crystallinity Index. Cellulose 2013, 20 (1), 583-588.

(97) Driemeier, C.; Calligaris, G. a. Theoretical and Experimental Developments for Accurate Determination of Crystallinity of Cellulose I Materials. J. Appl. Crystallogr. 2011, 44 (1), 184192.

(98) Ahvenainen, P.; Kontro, I.; Svedström, K. Comparison of Sample Crystallinity Determination Methods by X-Ray Diffraction for Challenging Cellulose I Materials. Cellulose 2016, 23 (2), 1073-1086.

(99) Edgar, C. D.; Gray, D. G. Influence of Dextran on the Phase Behavior of Suspensions of Cellulose Nanocrystals. Macromolecules 2002, 35 (19), 7400-7406.

(100) Bhattacharjee, S. DLS and Zeta Potential - What They Are and What They Are Not? J. Control. Release 2016, 235, 337-351.

(101) Garboczi, E. J.; Snyder, K. a.; Douglas, J. F.; Thorpe, M. F. Geometrical Percolation Threshold of Overlapping Ellipsoids. Phys. Rev. E 1995, 52 (1), 819-828.

(102) Boluk, Y.; Danumah, C. Analysis of Cellulose Nanocrystal Rod Lengths by Dynamic Light Scattering and Electron Microscopy. J. Nanoparticle Res. 2014, 16 (1), 2174.

(103) Maurer, R. J.; Sax, A. F.; Ribitsch, V. Moleular Simulation of Surface Reorganization and Wetting in Crystalline Cellulose I and II. Cellulose 2013, 20, 25-42.

(104) Lahiji, R. R.; Xu, X.; Reifenberger, R.; Raman, A.; Rudie, A.; Moon, R. J. Atomic Force Microscopy Characterization of Cellulose Nanocrystals. Langmuir 2010, 26 (6), 4480-4488. 
(105) Brinkmann, A.; Chen, M.; Couillard, M.; Jakubek, Z. J.; Leng, T.; Johnston, L. J. Correlating Cellulose Nanocrystal Particle Size and Surface Area. Langmuir 2016, acs.langmuir.6b01376.

(106) Postek, M. T.; Vladar, A.; Dagata, J.; Farkas, N.; Ming, B.; Wagner, R.; Raman, A.; Moon, R. J.; Sabo, R.; Wagner, T. H.; et al. Development of the Metrology and Imaging of Cellulose Nanocrystals. Meas. Sci. Technol. 2011, 22 (2), 24005-24015.

(107) Peng, Y.; Gardner, D. J.; Han, Y.; Kiziltas, A.; Cai, Z.; Tshabalala, M. a. Influence of Drying Method on the Material Properties of Nanocellulose I: Thermostability and Crystallinity. Cellulose 2013, 20 (5), 2379-2392.

(108) Langan, P.; Nishiyama, Y.; Chanzy, H. X-Ray Structure of Mercerized Cellulose II at $1 \AA$ Resolution. Biomacromolecules 2001, 2 (2), 410-416.

(109) Dorris, G. M.; Gray, D. G. The Surface Analysis of Paper and Wood Fibers by ESCA (Electron Spectroscopy for Chemical Analysis). I. Application to Cellulose and Lignin. Cellul. Chem. Technol. 1978, 12, 9-23.

(110) Johansson, L. S.; Campbell, J. M. Reproducible XPS on Biopolymers: Cellulose Studies. Surf. Interface Anal. 2004, 36 (8), 1018-1022.

(111) Edgar, C. D.; Gray, D. G. Smooth Model Cellulose I Surfaces from Nanocrystal Suspensions. Cellulose 2003, 10, 299-306.

(112) King, D. Oxidation of Gold by Ultraviolet Light and Ozone at 25 C. J. Vac. Sci. Technol. A Vac. ... 1995, No. October 1994, 1247-1253.

(113) Zoppe, J. O.; Habibi, Y.; Rojas, O. J.; Venditti, R. a; Johansson, L.-S.; Efimenko, K.; Österberg, M.; Laine, J. Poly(N-Isopropylacrylamide) Brushes Grafted from Cellulose Nanocrystals via Surface-Initiated Single-Electron Transfer Living Radical Polymerization. Biomacromolecules 2010, 11 (10), 2683-2691.

(114) Camarero Espinosa, S.; Kuhnt, T.; Foster, E. J.; Weder, C. Isolation of Thermally Stable Cellulose Nanocrystals by Phosphoric Acid Hydrolysis. Biomacromolecules 2013, 14 (4), 1223-1230.

(115) Hirai, A.; Inui, O.; Horii, F.; Tsuji, M. Phase Separation Behavior in Aqueous Suspensions of Bacterial Cellulose Nanocrystals Prepared by Sulfuric Acid Treatment. Langmuir 2009, 25 (1), 497-502.

(116) Honorato-Rios, C.; Kuhnhold, A.; Bruckner, J.; Dannert, R.; Lagerwall, J. P. Equilibrium Liquid Crystal Phase Diagrams and Detection of Kinetic Arrest in Cellulose Nanocrystal Suspensions. Front. Mater. 2016, 3 (21), 1-13.

(117) Kalashnikova, I.; Bizot, H.; Cathala, B.; Capron, I. Modulation of Cellulose Nanocrystals Amphiphilic Properties to Stabilize Oil/water Interface. Biomacromolecules 2012, 13 (1), 267275.

(118) Uetani, K.; Yano, H. Semiquantitative Structural Analysis of Highly Anisotropic Cellulose Nanocolloids. ACS Macro Lett. 2012, 1 (6), 651-655. 
(119) Giese, M.; Blusch, L. K.; Khan, M. K.; MacLachlan, M. J. Functional Materials from CelluloseDerived Liquid-Crystal Templates. Angew. Chemie - Int. Ed. 2015, 54 (10), 2888-2910.

(120) Niinivaara, E.; Faustini, M.; Tammelin, T.; Kontturi, E. Water Vapor Uptake of Ultrathin Films of Biologically Derived Nanocrystals: Quantitative Assessment with Quartz Crystal Microbalance and Spectroscopic Ellipsometry. Langmuir 2015, 31 (44), 12170-12176. 

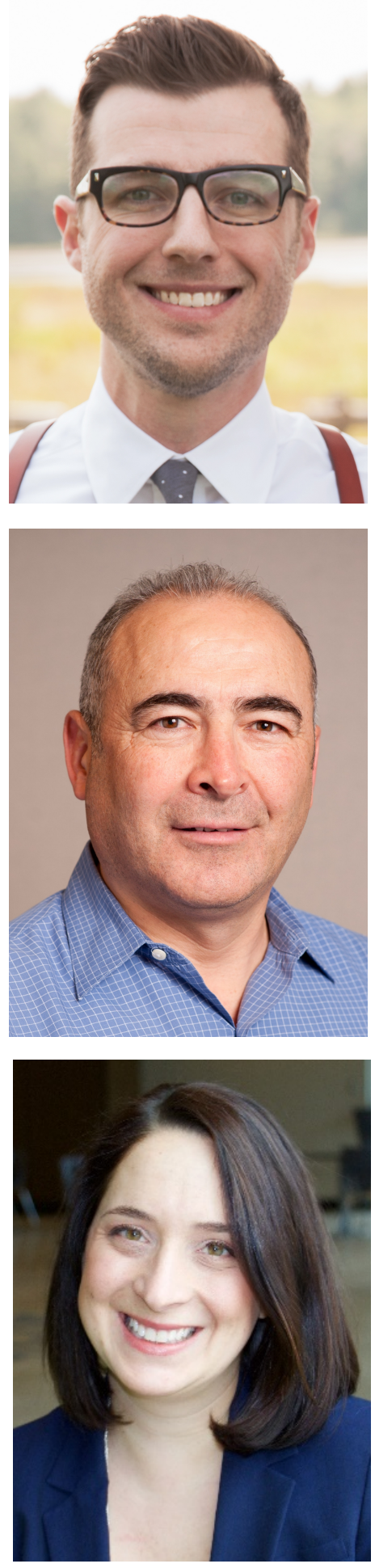

Michael S. Reid is a Ph.D. candidate in Chemical Engineering at McMaster University. He received his Honors B.Sc. degree in Chemical Physics (2009) from the University of Guelph and his M.Sc. in Chemistry (2012) from the University of Alberta. Since joining the Cranston research group his primary research interests include interfacial engineering, plant-based colloids and sustainable nanocomposite materials.

Marco Villalobos is the Director of Applications Development (Performance Materials \& Reinforcement Materials Division) at Cabot Corporation, and previously held R\&D manager positions at BASF, S.C. Johnson Polymer and was a research engineer at Monsanto, Mexico. His B.Sc. is in Chemical Engineering from the National University of Mexico, and he obtained both his M.Eng (1989) and Ph.D. (1993) in Chemical Engineering from McMaster University. His expertise is in the development and commercialization of new products and applications via market driven innovation with research interests in elastomers, plastics, composites, coatings, toners, adhesives, displays, inks, and batteries.

Emily D. Cranston is an Associate Professor in Chemical Engineering at McMaster University. She received her Honors B.Sc. degree in Chemistry at McGill University and her Ph.D. in Materials Chemistry (2008) in the group of Dr. Derek G. Gray, also at McGill University. She was a postdoctoral researcher at the Royal Institute of Technology in Sweden before returning to Canada in 2011. She is the Vice-Chair of the TAPPI Nanotechnology Division and recipient of the 2016 KINGFA Young Investigator's Award from the American Chemical Society's Cellulose and Renewable Materials Division. Cranston's research focuses on the design and characterization of sustainable nanocomposites and hybrid materials from cellulose and bio-based nanoparticles. 